\title{
Superresolution Mapping Using a Hopfield Neural Network With Fused Images
}

\author{
Minh Q. Nguyen, Peter M. Atkinson, and Hugh G. Lewis
}

\begin{abstract}
Superresolution mapping is a set of techniques to increase the spatial resolution of a land cover map obtained by softclassification methods. In addition to the information from the land cover proportion images, supplementary information at the subpixel level can be used to produce more detailed and accurate land cover maps. The proposed method in this research aims to use fused imagery as an additional source of information for superresolution mapping using the Hopfield neural network (HNN). Forward and inverse models were incorporated in the HNN to support a new reflectance constraint added to the energy function. The value of the function was calculated based on a linear mixture model. In addition, a new model was used to calculate the local endmember spectra for the reflectance constraint. A set of simulated images was used to test the new technique. The results suggest that fine spatial resolution fused imagery can be used as supplementary data for superresolution mapping from a coarser spatial resolution land cover proportion imagery.
\end{abstract}

Index Terms-Fused images, Hopfield neural network (HNN) optimization, soft classification, superresolution mapping.

\section{INTRODUCTION}

$\mathbf{T}$ HERE has been an increasing requirement for high spatial and spectral resolution remotely sensed imagery in a wide range of fields such as agriculture, urban planning, habitat management, and especially land cover mapping. To satisfy this requirement, several sensors launched recently provide images with a very high spatial resolution, such as IKONOS with 4-m multispectral (MS) and 1-m panchromatic (Pan) imagery and QuickBird with 2.6-m MS and 0.6-m Pan imagery [1]. However, the spatial detail in such imagery is still limited by the pixel, which represents the smallest element in a remotely sensed image. Conventionally, hard-classification approaches provide thematic maps at the pixel level, in which each pixel is assigned to just one class in the thematic map [2]. In most cases, the nature of the real landscape and the data acquisition process cause many "mixed pixels" in remotely sensed images [3]. If these mixed pixels are assigned to just one class as in hard classification, some information is lost.

Soft-classification approaches predict the proportional cover of each land cover class within each pixel. Several soft-classification approaches exist such as spectral mixture modeling [4],

Manuscript received October 9, 2004; revised October 17, 2005. The work of M. Nguyen was supported by a scholarship from the Ministry of Education and Training of Vietnam.

M. Q. Nguyen and P. M. Atkinson are with the Graduate School of Geography, University of Southampton, Southampton, SO17 1BJ, U.K. (e-mail: nguyenm@soton.ac.uk; pma@soton.ac.uk).

H. G. Lewis is with the Astronautics Research Group, School of Engineering Sciences, University of Southampton, Southampton, SO17 1BJ, U.K. (e-mail: H.G.Lewis@soton.ac.uk).

Digital Object Identifier 10.1109/TGRS.2005.861752 fuzzy $c$-means classifiers [5], artificial neural networks [6], [7], $k$-nearest neighbor classifiers [8], and support vector machines [9]. Soft classification produces a set of proportion images, and each of these images contains subpixel information on a given class. These images are more informative and appropriate depictions of land cover than those produced by the conventional hard classification. However, the location of the land cover classes in the mixed pixels is still unknown. In other words, the spatial resolution of the thematic map produced by soft classification is not increased relative to that of hard classification.

Superresolution mapping is a set of techniques for predicting the location of land cover classes within a pixel based on the proportion images produced by soft classification. Hence, superresolution mapping increases the spatial resolution of the resulting land cover maps. Since the concept of superresolution mapping was introduced by Atkinson [10], there have been several techniques proposed for superresolution mapping: spatial dependence maximization [10], subpixel per-field classification [11], linear optimization techniques [12], Hopfield neural network (HNN) optimization [13]-[17], two-point histrogram optimization [18], genetic algorithms [19], and feedforward neural networks [20]. These techniques are based on the concept of spatial dependence, which refers to the tendency of proximate subpixels to be more alike than those located far apart. In all these approaches, the detail and accuracy of the superresolution map were greater than the corresponding hard-classified images. However, these superresolution mapping methods have a limit to the detail and accuracy of the resulting thematic map since they were based only on the soft-classified proportion data at the pixel level and the spatial dependence assumption. It is, therefore, suggested that additional information could be useful.

An example of the inaccuracy of the above-mentioned superresolution mapping methods can be seen in Fig. 1. From the land cover proportion of a single class in Fig. 1(a), three possible resulting thematic maps at subpixel resolution can be obtained as in Fig. 1(b)-(d). All these maps satisfy the assumption of spatial dependence but the location of the subpixels in the central pixel is different. If some information can be provided at the subpixel level, then more accurate subpixel mapping results can be achieved.

The spatial resolution of thematic maps can be increased using raw remote sensing images without soft-classified data. Schneider and Steinwender [21] combined an image segmentation technique and spatial subpixel analysis based on the spatial pattern of pixels in a certain neighborhood to produce higher spatial resolution images. Further, Pinilla Ruiz and Ariza Lopez [22] used point spread function-derived deconvolution filters to increase the spatial resolution of multispectral images. 


\begin{tabular}{|c|c|c|}
\hline $100 \%$ & $100 \%$ & $100 \%$ \\
\hline $0 \%$ & $50 \%$ & $0 \%$ \\
\hline $100 \%$ & $100 \%$ & $100 \%$ \\
\hline
\end{tabular}

(a)

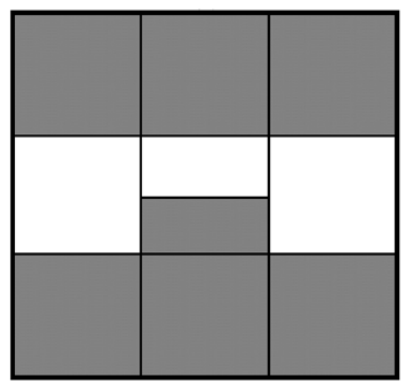

(c)

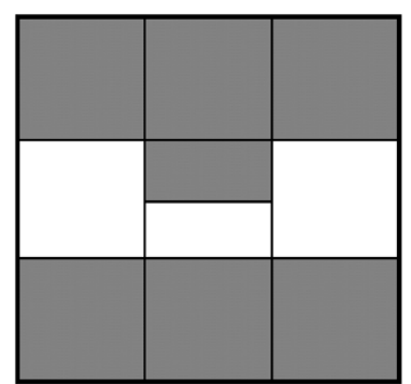

(b)

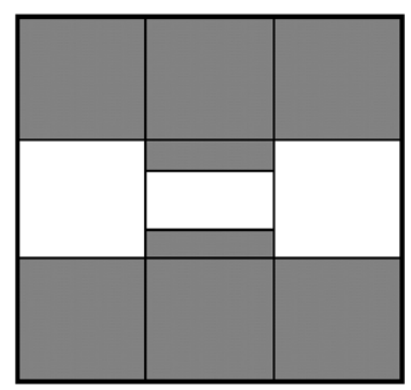

(d)
Fig. 1. (a) Land cover proportion image and (b), (c), (d) three possible resulting superresolution mapping images based on maximizing spatial dependence.

Image fusion is another approach to increase the spatial resolution of raw remotely sensed images by combining high spatial but low spectral resolution images with high spectral but low spatial resolution images to produce a high spectral and spatial resolution image. Amongst the approaches for fusing different data sources, the most common involves combining Pan and MS images to obtain a MS image at the spatial resolution of the Pan image. There have been several image fusion techniques such as intensity-hue-saturation (IHS) method [23], filter-based fusion [24], wavelets method [25], and Gram-Schmidt spectral sharpening [26]. For land cover classification, the fused MS image should preserve the spectral properties of the MS image at the spatial resolution of the Pan image. However, quality assessment for the fusion approaches proposed by Munechika et al. [27] and Wald et al. [28] showed that there is always spectral distortion in the fused image compared to the spectral properties of the original MS data.

There are two approaches for using a fused MS image for land cover classification. The fusion-then-classification approach uses the fused MS image directly for land cover classification. An example of the fusion-then-classification approach is given by Shackelford and Davis who used a 1-m fused MS image for urban mapping [29]. The classification-then-sharpening approach uses the fused MS image to sharpen a land cover proportion image obtained from the original MS image. The classification-then-sharpening approach was used by Foody to sharpen a fuzzy classification output [30] and by Gross and Schott to sharpen a proportion image obtained by spectral mixture analysis [31]. According to the results of both approaches, the accuracy of the thematic map using the fused MS image increased slightly in comparison with that of the original MS image. Evaluation of the two approaches by Robinson et al. [32] based on a linear mixing model indicated that the classification-then-sharpening approach was preferable in terms of

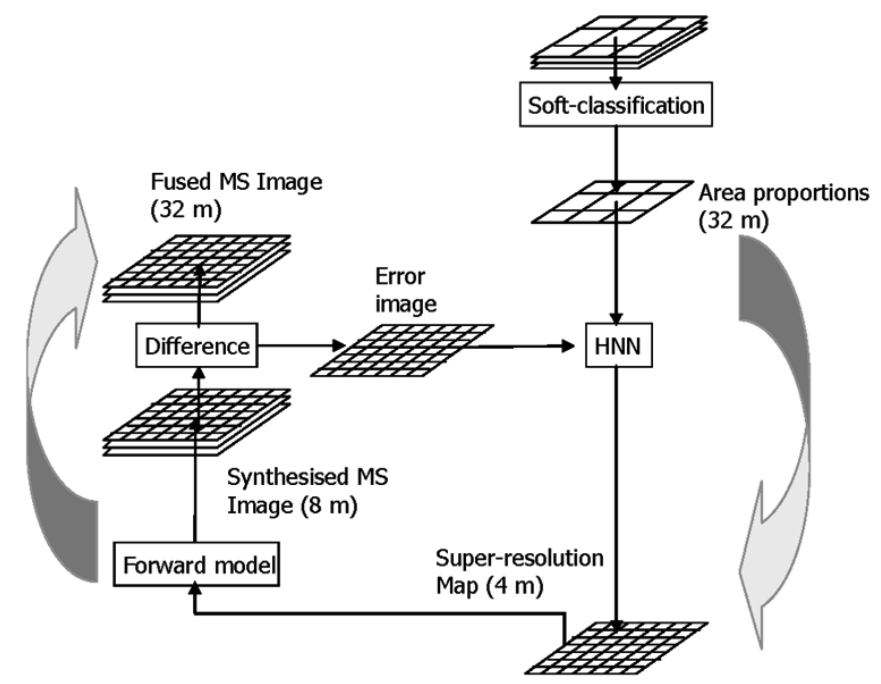

Fig. 2. HNN superresolution mapping using the fused multispectral mages.

accuracy. The fusion-then-classification approach produced a thematic map with lower accuracy due to the spectral distortion in the fused MS image.

\section{HOPFIELD NEURAL NETWORK}

This research aims to use a fused image as additional information for superresolution mapping. Theoretically, the fused MS image can provide useful information at an intermediate spatial resolution for predicting land cover at a finer spatial resolution. However, the spectral distortion of the fused images may cause some errors. To reduce the effect of spectral distortion, the classification-then-sharpening approach was incorporated into the HNN for superresolution mapping.

Fig. 2 is a graphical depiction of the proposed method to use a fused image for superresolution mapping by the HNN. From the MS images at the original spatial resolution the land cover area proportion images are produced by a soft-classification procedure. The area proportion images are then used to constrain the HNN to produce the superresolution land cover map in the first iteration of the optimization process. From the superresolution map at the first iteration, an estimated MS image is then produced using a forward model. The estimated MS image is then compared with the fused image and a reflectance error image is determined. For all neurons covered by the same pixel in the fused image, a value based on the reflectance error image is produced to adjust the estimated MS image. Thus, the HNN is constrained by the reflectance values of the fused image. The adjustment value, or reflectance constraint, along with the goal and constraint values in the HNN structure proposed by Tatem et al. [13], can be used in the optimization process for superresolution mapping by minimizing the energy function. After the optimization process, the estimated MS image produced by subpixels in the superresolution map should resemble the fused image.

The method presented is based on the structure of the HNN proposed by Tatem et al. [14], [15]. The structure of the HNN for superresolution mapping of two land cover classes can be seen in Fig. 3. A pixel at the original spatial resolution is divided into two interconnected matrices of neurons in the HNN. 


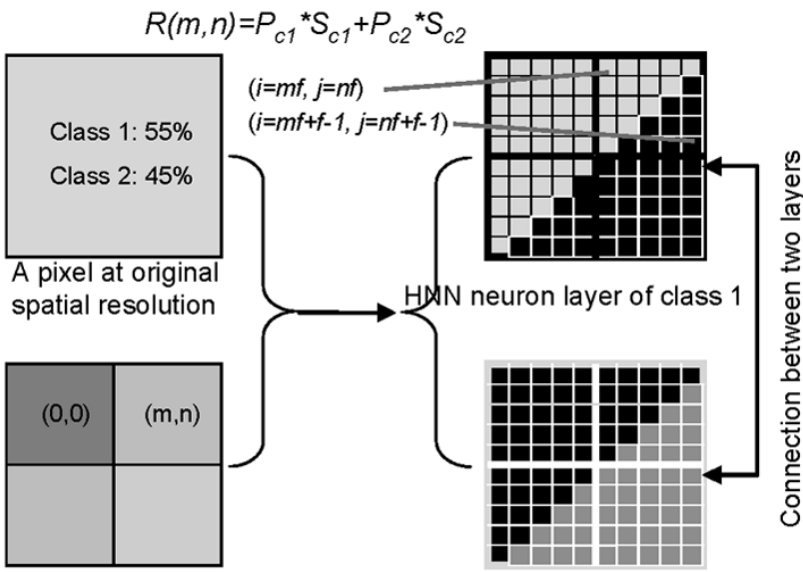

Corresponding four pixels at fusion spatial resolution

HNN neuron layer of class 2

Fig. 3. Reflectance constraint for subpixels covered by pixel $(m, n)$ at the fused level. A pixel at the original level contains four pixels at the fusion level. $m, n$ are the coordinates of the fused pixel. $R(m, n)$ is the reflectance value of the fused pixel $(m, n)$, and $S_{c 1}$ and $S_{c 2}$ are the endmember spectra of classes 1 and 2. $P_{c 1}$ and $P_{c 2}$ are average output values of the neurons of classes 1 and 2 that are covered by the fused pixel $(m, n) . f$ is the fusion factor.

Each neuron $(h, i, j)$ represents a subpixel at position $(i, j)$ in the land cover class $h$ and each matrix of neurons represents a land cover class. The HNN is a recurrent neural network and it reaches a stable state when the energy function is minimized. For superresolution mapping, the HNN is initialized using the soft-classified land cover proportions and runs until it converges to a monotonic stable state [13]. At the stable state, the output values of the neurons are binary values. If the output value of the neuron $(h, i, j)$ is 1 , the subpixel $(i, j)$ is assigned to the land cover class $h$. Otherwise, if the output value is 0 , the subpixel $(i, j)$ does not belong to the class $h$. The energy function can be expressed as

$$
E=-\sum_{h} \sum_{i} \sum_{j}\left(k_{1} G 1_{h i j}+k_{2} G 2_{h i j}+k_{3} P_{h i j}+k_{4} M_{h i j}\right)
$$

where $k_{1}, k_{2}, k_{3}$, and $k_{4}$ are weighting constants. Values of the weighting constants define the effects of the conresponding goal functions, proportion constraint and multiclass constraint to the energy function. For each neuron $(h, i, j), G 1_{h i j}$ and $G 2_{h i j}$ are the values of the spatial clustering or goal functions. The values of $G 1_{h i j}$ and $G 2_{h i j}$ can be determined by

$$
\frac{d G 1_{h i j}}{d v_{h i j}}=\frac{1}{2}\left(1+\tanh \left(\frac{1}{8} \sum_{\substack{d=i-1 \\ d \neq i}}^{i+1} \sum_{\substack{e=j-1 \\ e \neq j}}^{j+1} v_{h d e}-0.5\right) \lambda\right)\left(v_{h i j}-1\right)
$$$$
\frac{d G 2_{h i j}}{d v_{h i j}}=\frac{1}{2}\left(1+\left(-\tanh \left(\frac{1}{8} \sum_{\substack{d=i-1 \\ d \neq i}}^{i+1} \sum_{\substack{e=j-1 \\ e \neq j}}^{j+1} v_{h d e}\right) \lambda\right)\right)\left(v_{h i j}\right)
$$

where $\lambda$ is the gain or the steepness of the tanh function (usually assigned a value 100 [14]). $1 / 8 \equiv 1 / N$, where $N$ is the number of pixels in the neighborhood used in the goal function, 0.5 is the threshold, $v_{h i j}$ is the ouput value of the neuron $(h, i, j)$. The first goal function (2) is used to increase the output value $v_{h i j}$ of the neuron if the average output value of the eight surrounding neurons is greater than 0.5 . In contrast, the second goal function (3) decreases $v_{h i j}$ if the average output value of the eight surrounding neurons is less than 0.5 .

The value $P_{h i j}$ in (1) is the proportion constraint. This value retains the land cover proportion for each original pixel and is defined as

$$
\frac{d P_{h i j}}{d v_{h i j}}=\frac{1}{2 z^{2}} \sum_{d=x z}^{x z+z-1} \sum_{e=y z}^{y z+z-1}\left(1+\tanh \left(v_{h d e}-0.5\right) \lambda\right)-a_{h x y}
$$

where $1 / 2 z^{2} \sum_{d=x z}^{x z+z-1} \sum_{e=y z}^{y z+z-1}\left(1+\tanh \left(v_{h d e}-0.5\right) \lambda\right)$ is the estimated proportion and $a_{h x y}$ is the input proportion of the land cover $h$ of the pixel $(x, y)$ which is obtained by soft classification. The pixel $(x, y)$ is the corresponding pixel at the original spatial resolution to which the subpixel or neuron $(h, i, j)$ belongs. $z$ is the zoom factor, which determines the increase in spatial resolution from the original image to the superresolution mapping image. The proportion constraint function contributes a positive value if the estimated proportion of the class $h$ is greater than the input proportion. As a result, the network reduces the output values of neurons within the pixel $(x, y)$ in the class layer $h$. Conversely, if the estimated proportion is less than the input proportion, the proportion constraint produces a negative value to increase the output values of the neurons in the class $h$.

The multiclass value $M_{h i j}$ is used to reduce the output of the neurons if the sum of outputs of $c$ classes at the position $(i, j)$ is greater than 1 . If the sum of outputs of $c$ classes is less than 1 , the function increases the output of the neurons at the position $(i, j)$. The value of the multiclass constraint is calculated as

$$
\frac{d M_{h i j}}{d v_{h i j}}=\left(\sum_{k=0}^{c} v_{k i j}\right)-1
$$

To use the fused image for superresolution mapping by the HNN, the energy function in (1) is modified by adding a reflectance constraint. In this experiment, a function based on the reflectance of the fused image is added to the goal functions and proportion constraint that comprise the energy function. The new energy function for each band of the fused image can be expressed in an equation as follows:

$$
\begin{aligned}
E=-\sum_{h} \sum_{i} \sum_{j}\left(k_{1} G 1_{h i j}+k_{2} G 2_{h i j}+k_{3} P_{h i j}\right. & \\
& \left.+k_{4} M_{h i j}+k_{5} R_{h i j}\right)
\end{aligned}
$$

where $R_{h i j}$ is the reflectance constraint value for each neuron $(h, i, j)$.

The structure of the modified HNN can be seen in Fig. 3 . Each neuron in the HNN represents a subpixel point in the original spatial resolution image. The fusion factor $f$ determines the increase in spatial resolution of the new superresolution image in comparison with the fused image. Apart from the proportion constraint for each original pixel, $f \times f$ subpixels covered by pixel $(m, n)$ of the fused image are constrained by a reflectance constraint. The reflectance constraint is based on the principle 
that the average predicted reflectance from all subpixels located within a pixel of the fused image should be equal to the observed reflectance (or target reflectance) of that pixel.

For each band of the fused image, there is an additional constraint for the energy function. The energy function is minimized if the derivatives of variables in (6) converge to zero for each neuron $(h, i, j)$

$$
\begin{aligned}
\frac{d E_{h i j}}{d v_{h i j}}=k_{1} \frac{d G 1_{h i j}}{d v_{h i j}} & +k_{2} \frac{d G 2_{h i j}}{d v_{h i j}} \\
& +k_{3} \frac{d P_{h i j}}{d v_{h i j}}+k_{4} \frac{d M_{h i j}}{d v_{h i j}}+k_{5} \frac{d R_{h i j}}{d v_{h i j}} .
\end{aligned}
$$

The derivative values of $G 1, G 2, P$, and $M$ with respect to $v_{h i j}$ are computed using (2)-(5), respectively.

To derive the value $d R_{h i j} / d v_{h i j}$, the estimated reflectance $R_{B s m n}^{t}$ of the neurons representing the fused pixel $(m, n)$ can be defined by a forward model using a linear mixture model [4] as

$$
R_{B s m n}^{t}=V_{C 1} S_{B s C 1}+V_{C 2} S_{B s C 2}+\cdots+V_{C c} S_{B s C c}
$$

where the estimated proportion value $V_{C e}=$ $1 / f^{2}\left(\sum_{p=m f}^{(m f+f-1)} \sum_{q=n f}^{(n f+f-1)} v_{C e p q}\right)$ and $S_{B s C e}$ is the endmember spectra of the land cover class $C e$ for a spectral band $B s$.

Similarly, the observed reflectance for pixel $(m, n)$ can be expressed using the same forward model as

$$
R_{B s m n}^{o}=P_{C 1} S_{B s C 1}+P_{C 2} S_{B s C 2}+\cdots+P_{C c} S_{B s C c}
$$

where $R_{B s m n}^{o}$ is the observed reflectance of pixel $(m, n)$ in a fused image band, $c$ is the total number of land cover classes, $P_{C 1}, P_{C 2}, \ldots, P_{C c}$ are the proportions of a given land cover class covered by a pixel of the fused images $\left(\sum P=1\right)$, and $S_{B s C 1}, S_{B s C 2}, \ldots, S_{B s C c}$ are endmember spectra of the land cover classes $C 1, C 2, \ldots, C c$ in a MS fused band $B s$.

From (8) and (9), the difference between the observed and estimated reflectance of the neurons representing the fused pixel $(m, n)$ is defined by

$$
R^{o}-R^{t}=\sum_{e=1}^{c} P_{C e} S_{B s C e}-\sum_{e=1}^{c} V_{C e} S_{B s C e} .
$$

The value of the reflectance constraint requires that the reflectance difference in (10) should be zero for every spectral band. Accordingly, the estimated proportion value $V_{C e}$ is expected to converge to the land cover proportion $P_{C e}$ within each pixel of the fused image. Therefore, the value of the reflectance constraint for the fused pixel $(m, n)$ can be produced based on an inverse model using the linear mixture model equation

$$
R_{m n}^{o}=S P_{m n}
$$

where $\mathbf{R}_{m n}^{o}=\left[R_{B 1}{ }^{o} \ldots R_{B s}{ }^{o} \ldots R_{B b}{ }^{o}\right]_{m n}^{T}$ is the vector of reflectance values of the fused spectral bands $B 1, \ldots, B s, \ldots$, $B b$ and

$$
\mathbf{S}=\left[\begin{array}{lll}
S_{B 1 C 1} & \cdots & S_{B 1 C c} \\
S_{B s C 1} & \cdots & S_{B s C c} \\
S_{B b C 1} & \cdots & S_{B b C c}
\end{array}\right]
$$

is the matrix of endmember spectra values for $b$ fused spectral bands and $\mathbf{P}$ is the vector of land cover proportion values.

Using the least squares linear mixture model, land cover class proportions of the fused pixel $(m, n)$ can be predicted by

$$
\mathbf{P}_{m n}=\left(\mathbf{S}^{\mathbf{T}} \mathbf{S}\right)^{-1} \mathbf{S}^{\mathbf{T}} \mathbf{R}_{m n}^{\circ}=\mathbf{M} \mathbf{R}_{m n}^{\circ} \quad \text { with } \mathbf{M}=\left(\mathbf{S}^{\mathbf{T}} \mathbf{S}\right)^{-1} \mathbf{S}^{\mathbf{T}}
$$

From the (13), the values for reflectance constraints for all fused spectral bands are calculated from the difference between observed and estimated reflectance of $b$ fused multispectral bands as

$$
\begin{aligned}
\Delta & =\left[\begin{array}{c}
d R / d v_{C 1 i j} \\
\ldots \\
d R / d v_{C c i j}
\end{array}\right]=\left[\begin{array}{c}
P_{C 1} \\
\ldots \\
P_{C c}
\end{array}\right]-\left[\begin{array}{c}
V_{C 1} \\
\ldots \\
V_{C c}
\end{array}\right] . \\
& =\mathbf{M}\left(\mathbf{R}_{m n}^{\circ}-\mathbf{R}_{m n}^{t}\right)=M\left[\begin{array}{c}
R_{B 1 m n}^{\circ}-R_{B 1 m n}^{t} \\
\cdots \\
R_{B b m n}^{\circ}-R_{B b m n}^{t}
\end{array}\right] .
\end{aligned}
$$

If the number of fused image spectral bands is less than the number of land cover classes, the value of the reflectance constraint cannot be known.

\section{EXAMPLE 1: SiMULATED IKONOS IMAGE}

\section{A. Data}

In this experiment, a set of data based on IKONOS imagery was used. The set of data is a simulated 32-m MS image and an 8-m fused spectral image created by degrading a real IKONOS image. The ratio between the spatial resolution of the simulated MS and fused images is similar to the ratio between the real 4-m MS and 1-m panchromatic (PAN) IKONOS images. Thus, the algorithm should be applicable to the real imagery (i.e., 4-m MS and 1-m fused image) if it performs successfully on the simulated imagery. There are two reasons for using simulated imagery. Firstly, it was possible to evaluate the quality of the fused imagery. Secondly, the simulation ensured that there were no errors in image registration between the reference image and the land cover image obtained by superresolution mapping. For the simulated IKONOS image, the experiment was implemented in four steps as follows: (A) Raw data analysis, (B) Data simulation, (C) Preprocessing, and (D) Superresolution mapping (Fig. 4).

\section{1) Raw Data Analysis:}

Raw Data: An IKONOS MS image was acquired over Eastleigh and Chandler Ford, Southampton, U.K. The IKONOS image consisted of four 4-m MS bands in the following wavebands: red $(632-698 \mathrm{~nm})$, near-infrared (NIR: $757-853 \mathrm{~nm}$ ), green $(506-595 \mathrm{~nm})$, and blue $(445-516 \mathrm{~nm})$ and a 1-m PAN band (450-900 nm). Based on image fusion, the MS and PAN bands can be used to produce four fused MS image bands at 1-m spatial resolution.

Reference Data and Statistical Information: The experiment was implemented in an area of $64 \times 64$ pixels (at 4-m spatial resolution) that consisted of three land cover classes: cereal, grass, and trees [Fig. 5(a)-(d)]. These three land cover classes were produced using maximum-likelihood classification of the real IKONOS image. Statistical information such as the means 


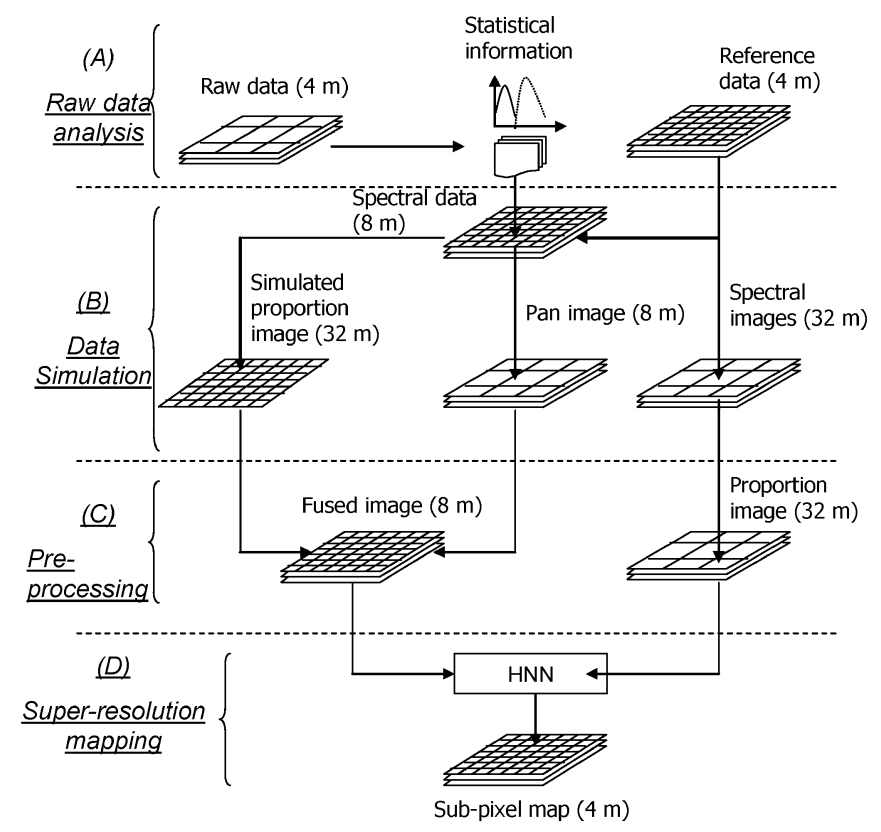

Fig. 4. Four steps in experiment: (A) Raw data analysis, (B) Data simulation, (C) Preprocessing, and (D) Superresolution mapping.

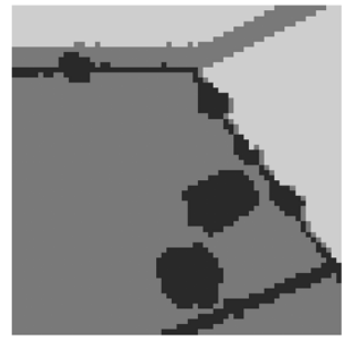

(a)

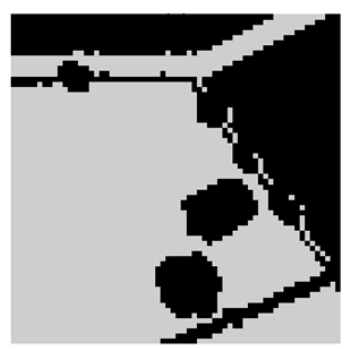

(c)

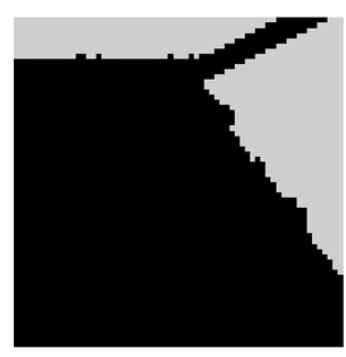

(b)

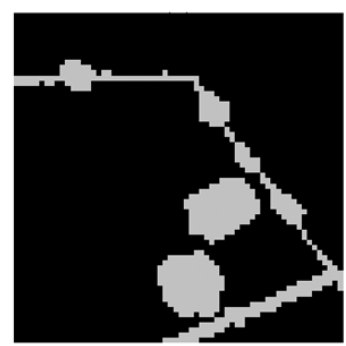

(d)
Fig. 5. (a) Land cover map at 4-m spatial resolution used for simulating data, (b) 4-m cereal class map, (c) 4-m grass class map, and (d) 4-m trees class map.

and standard deviations of the three land cover classes in the area was obtained (Table I and Fig. 6). The three land cover classes were used as a reference for the subpixel map obtained by the proposed algorithm.

2) Data Simulation:

Multispectral Imagery $(8 \mathrm{~m})$ : From the land cover map [Fig. 5(b)-(d)] at 4-m spatial resolution, a set of multispectral images at 4-m spatial resolution was simulated based on the random normal distribution and the mean and variance of each land cover class shown in Table I. The simulated MS image, therefore, is similar spectrally to a multispectral IKONOS image at 4-m spatial resolution. A MS image at 8-m spatial resolution was generated by degrading the 4-m simulated MS image by a
TABLE I

Statistical INFORMATION FOR THE CEREAL, GRASS, AND TREes Classes IN THE IKONOS IMAGE

\begin{tabular}{lllll}
\hline \hline Cereal class & & & & \\
\hline Band & Min & Max & Mean & $\begin{array}{l}\text { Standard } \\
\text { deviation } \\
1\end{array}$ \\
236 & 461 & 386.986 & 27.520 \\
2 & 336 & 574 & 491.683 & 27.282 \\
3 & 275 & 432 & 384.641 & 18.565 \\
4 & 257 & 346 & 322.755 & 9.993 \\
\hline Grass class & & & & \\
\hline \multirow{2}{*}{ Band } & Min & Max & Mean & Standard \\
1 & 220 & 325 & 259.636 & 12.303 \\
2 & 377 & 623 & 492.909 & 29.851 \\
3 & 283 & 354 & 314.681 & 7.864 \\
4 & 271 & 309 & 289.369 & 4.618 \\
\hline Trees class & & & & \\
\hline \multirow{2}{*}{ Band } & Min & Max & Mean & Standard \\
1 & 141 & 238 & 183.983 & 25.127 \\
2 & 194 & 713 & 421.699 & 105.667 \\
3 & 218 & 311 & 259.706 & 20.061 \\
4 & 244 & 286 & 264.162 & 8.887 \\
& & & & \\
\hline \hline
\end{tabular}

factor of two to produce [Fig. 7(a)-(d)]. These images were used as reference to evaluate the quality of the fused image produced below.

Panchromatic Imagery $(8 \mathrm{~m})$ : The $8-\mathrm{m}$ simulated MS image was then used to create a simulated PAN image [Fig. 7(e)] based on a simple spectral convolution of the blue, green, red, and NIR bands of the 8-m simulated MS image (the wavelength of the PAN band of the IKONOS image covers these four bands) as

$$
\operatorname{Pan}=\frac{\text { BLUE }+ \text { GREEN }+ \text { RED }+ \text { NIR }}{4} .
$$

Multispectral Imagery $(32 \mathrm{~m})$ : The 32-m MS image [Fig. 7(f)-(i)] was produced by degrading the 4-m MS image by a factor of eight. The 32-m MS image was then used for soft classification and image fusion to produce a 32-m land cover proportion image and an 8-m fused image.

3) Preprocessing:

Fused Imagery $(8 \mathrm{~m})$ : Amongst the four fused spectral bands, three were used in the experiment (blue, green, and red). The NIR band of the fused image was not used because of the scattered distribution and the spectral (Fig. 6) overlap of all three land cover classes over this band. From the simulated 8-m PAN and 32-m MS images, the 8-m fused image [Fig. 7(k)-(m)] was predicted using the Gram-Schmidt Spectral Sharpening method [26]. The fused image was evaluated based on the root mean square (RMS) error for each band [27]. The RMS errors of the red, green, and blue bands were 15.74 digital number (DN), 8.27 $\mathrm{DN}$ and 5.44 DN, respectively. Comparing with the RMS errors of the fused image obtained in Munechika et al. [27], the fused image produced was similar to that of real data.

Simulated Land Cover Proportion Imagery $(8 \mathrm{~m})$ : To provide a realistic test, a set of proportion images was produced using soft classification of the simulated 32-m MS image. The simulated MS image was used because in the simulated case the 


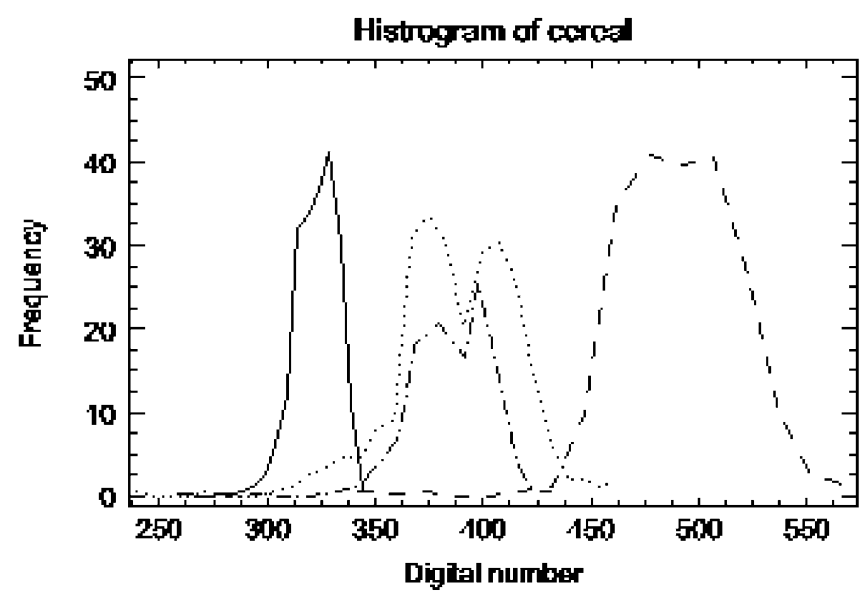

Histrogram of grass

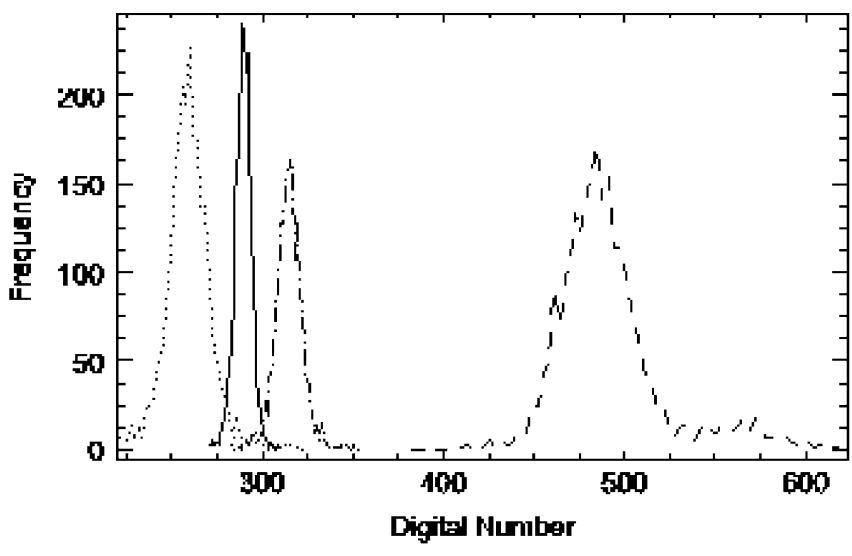

Histrogam of trops

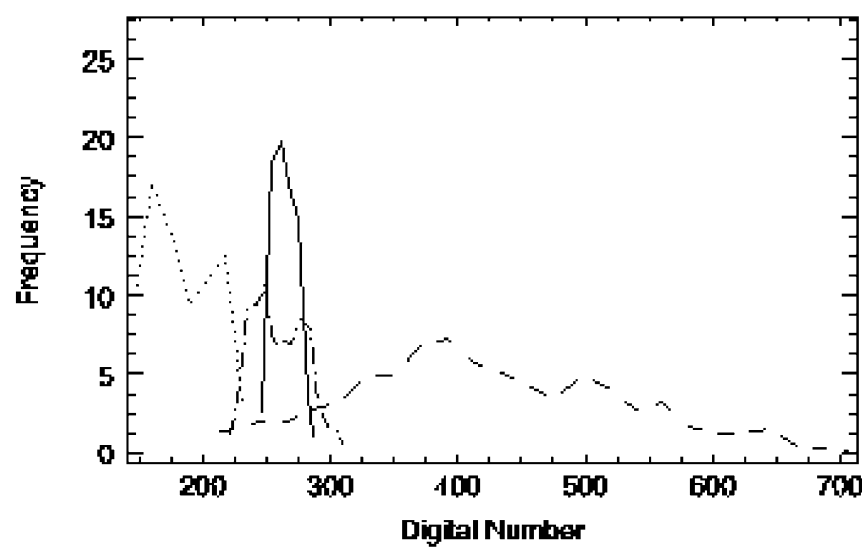

Fig. 6. Histrogram of three classes in four bands of IKONOS MS image. (Dotted line) Band 1. (Dashed line) Band 2. (Dotted and dashed line) Band 3. (Solid line) Band 4.

three land cover classes at the subpixel $(4 \mathrm{~m})$ level are known, facilitating direct evaluation of the technique. A $k$-nearest neighbor classifier $(k-\mathrm{NN})$ [8] was used for soft classification with $k=5$. The land cover proportion image was produced with overall area error proportion of $0.5552 \%$ and overall RMS error of 0.083775 pixels [13]. Statistics for the resulting land cover map from soft classification show that the land cover proportion images contained an amount of error similar to that of a soft-classified real MS image. In this sense, the simulated land cover proportion image was similar to that which might be obtained from real data.

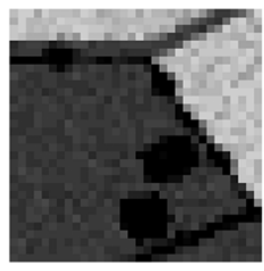

(a)

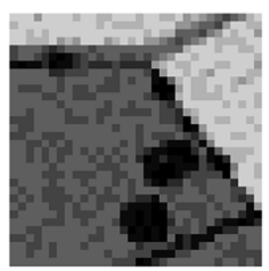

(d)

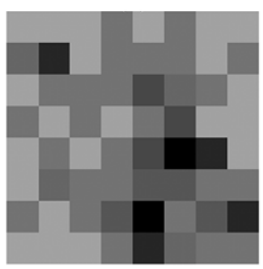

(g)

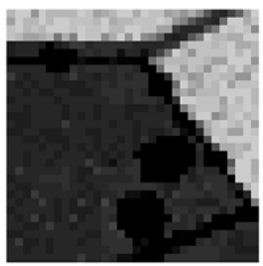

(k)

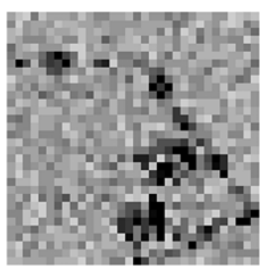

(b)

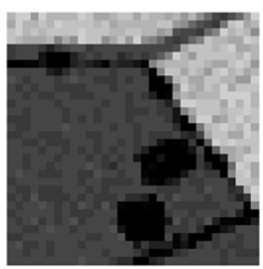

(e)

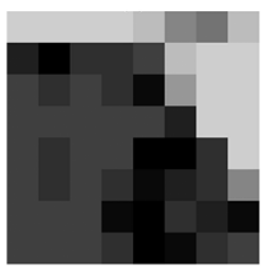

(h)

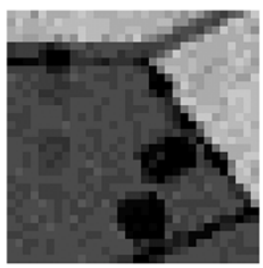

(1)

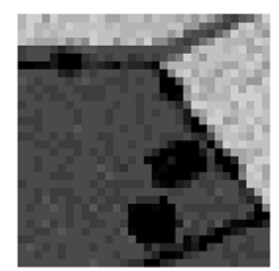

(c)

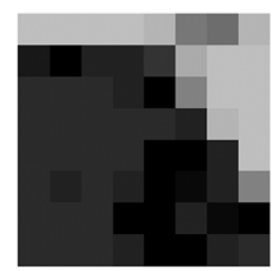

(f)

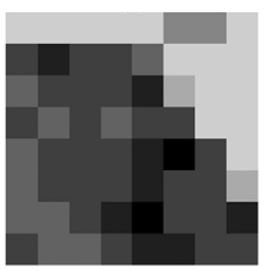

(i)

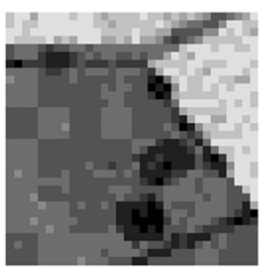

(m)
Fig. 7. Four bands (a) red, (b) NIR, (c) green, and (d) blue $8 \mathrm{~m}$ of simulated MS IKONOS image. (e) Eight-meter simulated PAN image. Four bands (f) red, (g) NIR, (h) green, and (i) blue of 32-m simulated image. Three bands (k) red, (l) green, and (m) blue of 8-m simulation of the fused MS image.

4) Global Endmember Spectra: The endmember spectra values in (7) can be acquired from laboratory measurements or can be estimated from training data [4]. Since the simulated MS image was created from statistics on the cereal, grass, and trees classes, the endmember spectra of these classes should be the means of the spectral distributions in the blue, green, and red bands.

5) Local Endmember Spectra: Three land cover classes exhibited a large variance over all four spectral bands (Table I and Fig. 6). Thus, the single set of endmember spectra values used in (2) was not appropriate for every pixel in the image. Investigation of the real IKONOS image indicated that the digital numbers of adjacent pixels of the same class were similar. Hence, it was suggested that using locally defined endmember spectra would be more appropriate for determining the local reflectance constraint value than using a single value for the whole image.

Local endmember spectra were produced from the land cover proportion image and the original MS image (e.g., 32-m land cover proportion image and 32-m MS image). Fig. 8 describes the local endmember spectra estimation process. The endmember spectra of the pixel $(m, n)$ of a given $8-m$ fused spectral band can be defined based on the class proportions and 


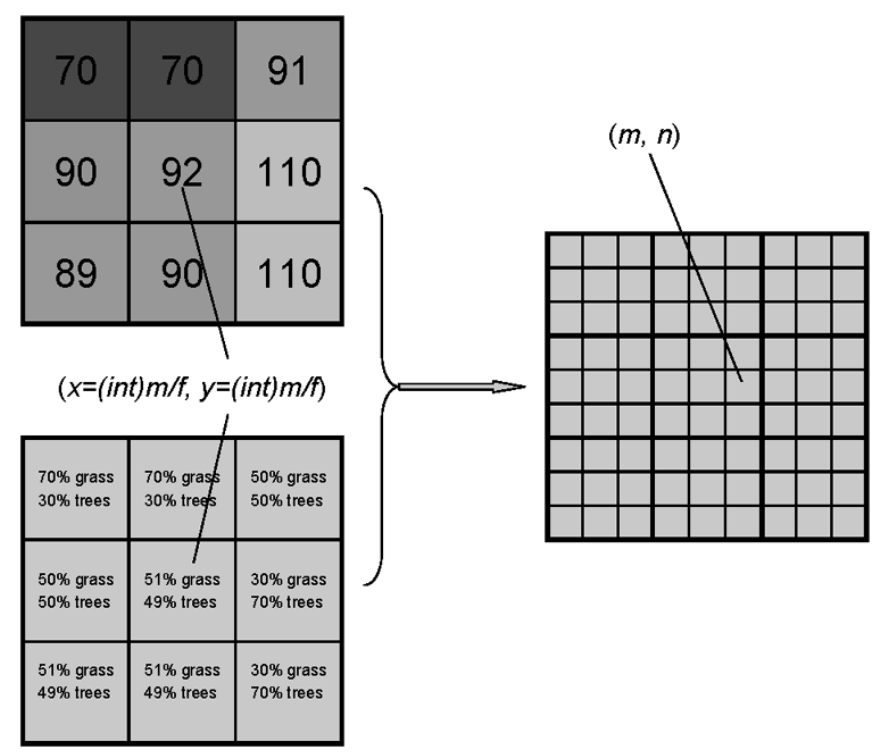

Fig. 8. Local endmember spectra calculation. $(m, n)$ are coordinates of the fused image pixel. and $(x, y)$ are coordinates of the pixel in the original image that corresponds to the fused pixel $(m, n)$. From land cover proportion and digital number of pixel $(x, y)$ and its eight surrounding pixels, the local spectra of the pixel $(m, n)$ can be calculated.

the reflectance value of the corresponding pixel $(x, y)$ and its eight surrounding pixels of the same spectral band of the 32-m MS image. For each spectral band and each pixel $(x, y)$, an equation exists as follows:

$$
R_{B i}^{x y}=S_{B i C 1} P_{C 1}^{x y}+S_{B i C 2} P_{C 2}^{x y}+\cdots+S_{C c} P_{C c}^{x y}
$$

where $R_{B i}^{x y}$ is the digital number of pixel $(x, y)$ in spectral band $B_{i}, P_{C 1}^{x y}, P_{C 2}^{x y}, \ldots, P_{C c}^{x y}$ are class proportions and $S_{B i C 1}, S_{B i C 2}, \ldots, S_{B i C c}$ are the local endmember spectra of the pixel $(x, y)$ in spectral band $B_{i}$.

With eight surrounding pixels, there are eight equations which can be rewritten in matrix form as

$$
\mathbf{R}_{B i}^{x y}=\mathbf{P S}_{B i}
$$

where $R_{B i}^{x y}=\left[R_{B i}^{(x-1)(y-1)} \ldots R_{B i}^{(x+1)(y+1)}\right]^{T}, S_{B i}^{x y}=$ $\left[S_{B i C 1} \cdots S_{B i C c}\right.$, and

$$
P=\left[\begin{array}{ccc}
P_{C 1}^{(x-1)(y-1)} & \ldots & P_{C c}^{(x-1)(y-1)} \\
\ldots & \ldots & \ldots \\
P_{C 1}^{x y} & \ldots & P_{C c}^{x y} \\
\cdots & \cdots & \cdots \\
P_{C 1}^{(x+1)(y+1)} & \ldots & P_{C c}^{(x+1)(y+1)}
\end{array}\right]
$$

Using the least squares method, the local endmember spectra $S_{B i}$ can be resolved as

$$
\mathbf{S}_{B i}=\left(\mathbf{P}^{T} \mathbf{P}\right)^{-1} \mathbf{P}^{T} \mathbf{R}_{B i}
$$

Amongst the pixels that are used to determine the local endmember spectra, the pixel $(x, y)$ should be the most important since it covers the fused pixel $(m, n)$. To emphasize the contribution of the corresponding pixel $(x, y)$ to the endmember spectra, a weight mechanism was used such that (19) becomes

$$
\mathbf{S}_{B i}=\left(\mathbf{P}^{T} \mathbf{W} \mathbf{P}\right)^{-1} \mathbf{W} \mathbf{P}^{T} \mathbf{R}_{B i}
$$

where $W$ was the diagonal matrix

$$
W=\left[\begin{array}{ccccc}
w^{(x-1)(y-1)} & 0 & 0 & 0 & 0 \\
0 & \cdots & 0 & 0 & 0 \\
0 & 0 & w^{x y} & 0 & 0 \\
0 & 0 & 0 & \cdots & 0 \\
0 & 0 & 0 & 0 & w^{(x+1)(y+1)}
\end{array}\right]
$$

and $w^{(x-1)(y-1)}, \ldots, w^{x y}, \ldots, w^{(x+1)(y+1)}$ are weight values for each corresponding pixel. The assumption was tested using the weight value $w^{x y}$ of 1 up to 20 and the other weight values of 1 . The optimal weight value $w^{x y}$ was determined based on the Kappa index of agreement (KIA) $\kappa$ of results of the superresolution mapping using the fused image.

\section{B. Results}

Two sources of data were used in superresolution mapping using fused imagery. The first data source was the land cover proportion image obtained by soft classification. The second data source was the fused image. In the experiment using the simulated land cover proportion image, both data sources contained an amount of error similar to that of real data.

From the predicted soft-classified land cover proportion image [Fig. 9(a)-(c)], the 4-m subpixel land cover maps were obtained using the traditional HNN [Fig. 9(g)-(i)], the HNN using the fused image with global endmember spectra [Fig. 9(j)-(1)] and the HNN using the fused image with local endmember spectra [Fig. 9(m)-(o)]. The greatest accuracy land cover map was obtained with the weighting coefficients of $k_{1}=70, k_{2}=70, k_{3}=70, k_{4}=70$, and $k_{5}=70$ after 6000 iterations and the optimal weight value of 14 to determine the local spectra. The 32-m hard-classified land cover image [Fig. 9(d)-(f)] was produced from the 32-m multispectral image [Fig. 9(g)-(j)] using a neural network. Accuracy statistics for each class based on KIA, overall accuracy, and per-class omission and commission errors are presented in Table II to evaluate the predicted subpixel map.

The map produced by the new HNN superresolution technique with real proportion image data was more accurate than the hard classification and traditional HNN in retaining small and linear objects. Despite the effect caused by the error in the class proportion image, the linear features in the trees class were recreated by both the new HNN with global and local endmember spectra. However, the errors from soft classification caused some artefacts in the trees class when the new HNN with global endmembers was used. These artefacts can be seen when comparing Figs. 7(d) and 9(1). The artefacts occurred mostly for pixels where the soft classification predicted some erroneous land cover proportions [the erroneous proportions can be seen clearly in Fig. 9(b)]. The artefacts did not occur in the resulting land cover map produced by the HNN with local endmembers. This can be explained as follows: the use of global endmembers led to an inability to reduce the effects of the erroneous proportions locally in some cases.

The statistics in Table II showed considerable increase in all accuracy values for the new HNN technique in comparison with the hard-classification and traditional HNN. The overall accuracy increased from $86.52 \%$ for the hard classification and $88.53 \%$ for the traditional HNN to $87.92 \%$ for the new HNN 


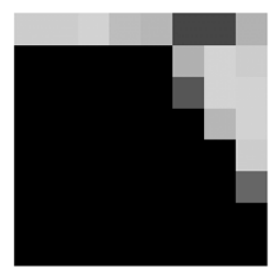

(a)

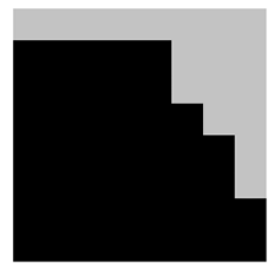

(d)

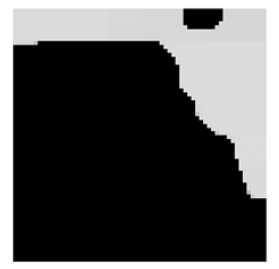

(g)

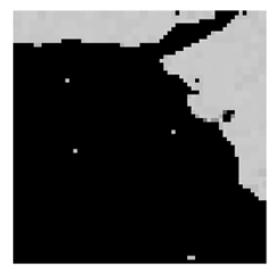

(j)

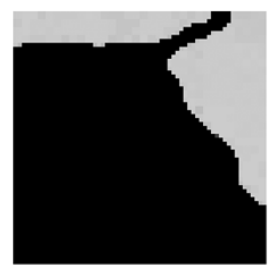

(m)

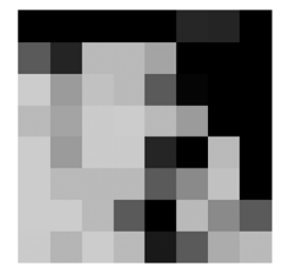

(b)

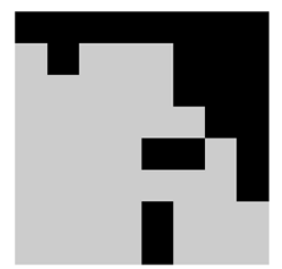

(e)

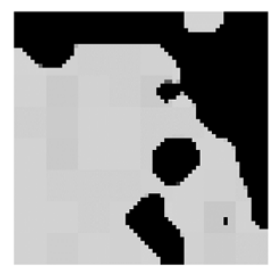

(h)

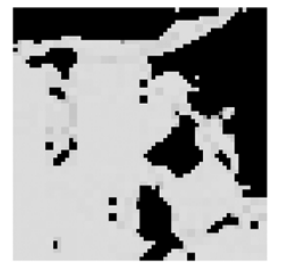

(k)

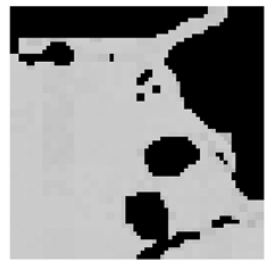

(n)

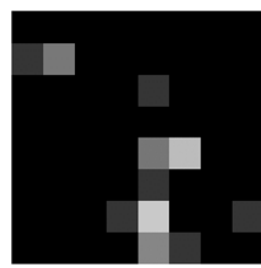

(c)

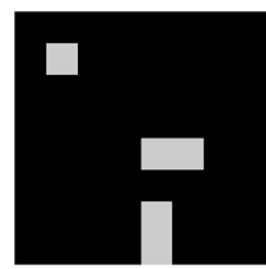

(f)

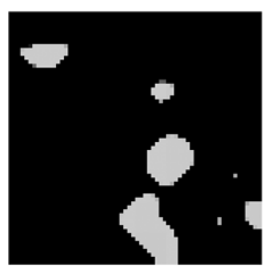

(i)

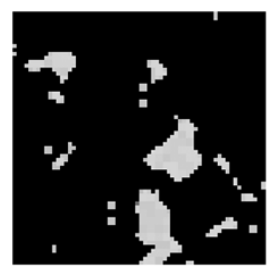

(1)

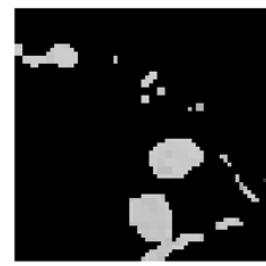

(o)
Fig. 9. (a) Four-meter Cereal, (b) Grass, and (c) Trees land cover proportion image. (d) Four-meter Cereal, (e) Grass, and (f) Trees hard-classified land cover image. (g) Four-meter Cereal, (h) Grass, (i) Trees HNN superresolution mapping image. (j) Four-meter Cereal, (k) Grass, and (1) Trees HNN superresolution mapping using the fused image with the global endmember spectra resulting image. (m) Four-meter Cereal, (n) Grass, and (o) Trees HNN superresolution mapping using the fused image with the local endmember spectra resulting image.

with global endmembers and $91.19 \%$ for the new HNN with local endmembers. The visual and statistical improvement of the resulting subpixel maps when using a real proportion image showed that the new algorithm can increase the accuracy of the thematic mapping with the real image data if the image registration error is not taken in to account.

Similar to the visual comparison, the statistics highlighted a problem with the new HNN with global endmembers. This problem resulted in an increase in the commission error for the trees class from $20.69 \%$ for the traditional $\mathrm{HNN}$ to $30.02 \%$ for the new HNN with global endmembers. Similarly, the omission
TABLE II

ACCURACY STATISTICS OF SimULATED IKONOS RESUltS

\begin{tabular}{|c|c|c|c|c|c|}
\hline \multicolumn{6}{|c|}{ Statistics for the hard classified image } \\
\hline & Cereal & Grass & Trees & ErrorO (\%) & ErrorC (\%) \\
\hline Unclassified & 0 & 0 & 0 & & \\
\hline Cereal & 1005 & 126 & 21 & 3.74 & $12.76 \%$ \\
\hline Grass & 39 & 2305 & 280 & 8.42 & $12.16 \%$ \\
\hline Trees & 0 & 86 & 234 & 56.26 & $26.88 \%$ \\
\hline \multicolumn{2}{|c|}{$\mathrm{KIA}-\kappa=$} & 0.7430 & \multicolumn{2}{|c|}{ Overall accuracy $=$} & $86.52 \%$ \\
\hline
\end{tabular}

Statistics for the HNN super-resolution mapping without using the fused image

\begin{tabular}{lllllc}
\hline & Cereal & Grass & Trees & ErrorO $(\%)$ & ErrorC $(\%)$ \\
Unclassified & 0 & 4 & 3 & & 1.000 \\
Cereal & 977 & 89 & 2 & 4.60 & $8.52 \%$ \\
Grass & 63 & 2350 & 231 & 8.14 & $11.12 \%$ \\
Trees & 4 & 74 & 299 & 36.82 & $20.69 \%$ \\
& KIA $-\kappa=$ & 0.7814 & Overall accuracy $=$ & $88.53 \%$ \\
\hline
\end{tabular}

Statistics for the HNN super-resolution mapping using the fused image (Global spectra)

$\begin{array}{llllll} & \text { Cereal } & \text { Grass } & \text { Trees } & \text { ErrorO (\%) } & \text { ErrorC (\%) } \\ \text { Unclassified } & 11 & 4 & 2 & & 1.0000 \\ \text { Cereal } & 931 & 39 & 0 & 10.82 & 4.02 \\ \text { Grass } & 91 & 2346 & 209 & 6.79 & 11.34 \\ \text { Trees } & 11 & 128 & 324 & 39.44 & 30.02 \\ \text { Total } & 1044 & 2517 & 535 & & \\ & \text { KIA }-\kappa= & 0.7897 & \text { Overall accuracy }= & 87.92 \%\end{array}$

Statistics for the HNN super-resolution mapping using the fused image (Local spectra value of 14)

\begin{tabular}{llllll}
\hline & Cereal & Grass & Trees & ErrorO $(\%)$ & ErrorC $(\%)$ \\
Unclassified & 6 & 0 & 0 & & 1.000 \\
Cereal & 994 & 39 & 0 & 4.79 & 3.68 \\
Grass & 38 & 2412 & 206 & 4.17 & 9.19 \\
Trees & 6 & 67 & 329 & 38.50 & 18.16 \\
& KIA $-\kappa=$ & 0.8320 & Overall accuracy $=$ & $91.19 \%$ \\
\hline \hline
\end{tabular}

error obtained for the trees class by the traditional HNN superresolution mapping increased from $36.82 \%$ to $39.44 \%$ for the new HNN with global endmembers. Due to the problem with the erroneous proportion, the overall accuracy of the new HNN with global endmembers decreased just slightly in comparison with the map obtained with the HNN superresolution mapping, with the overall accuracy decreasing from $88.52 \%$ to $87.92 \%$. With local endmembers, the HNN using the fused image can resolve the problem that occurred when using a single set of global endmembers. The overall accuracy of the resulting map produced by the new HNN with the local endmembers increased greatly by approximately $3 \%$ to $91.19 \%$.

\section{EXAMPLE 2: DEGRADED QUICKBIRD IMAGE}

\section{A. Data}

Although simulated imagery provides greater control than real imagery for evaluating new algorithms, a common criticism is that simulated imagery may not provide a realistic test, primarily because image registration error is not included in the data. Therefore, to provide a more realistic test, and to address such concerns, a second set of proportion images was produced using a degraded QuickBird MS and PAN image. A degraded (rather than real) image was used because in the degraded case the three land cover classes at the subpixel level are known, facilitating direct evaluation of the impact of image registration error on the technique. 


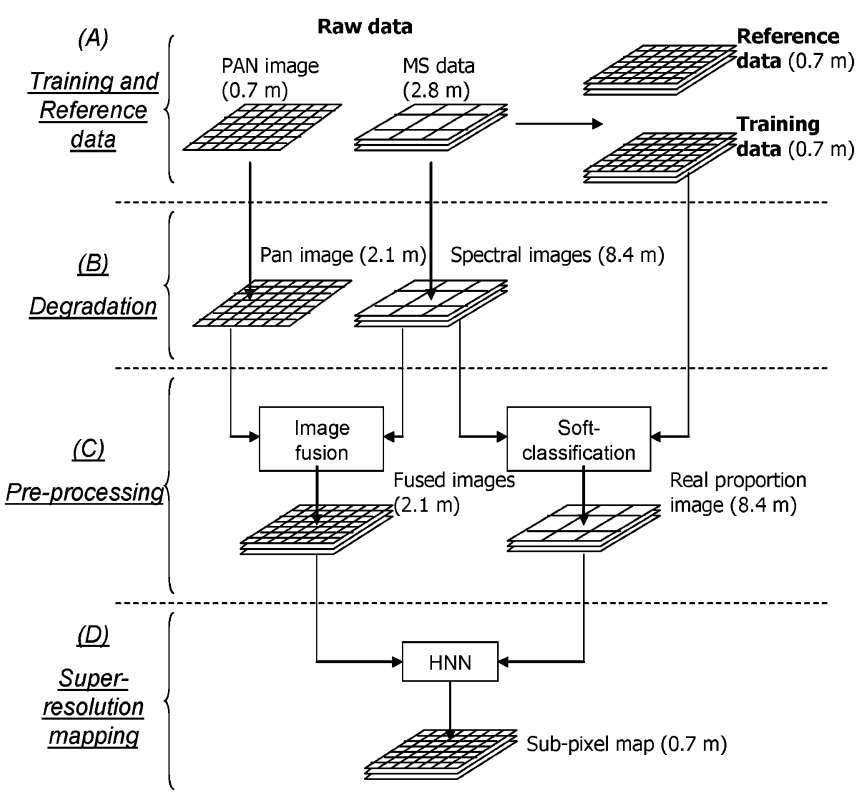

Fig. 10. Four steps in experiment. (A) Raw data analysis. (B) Data simulation. (C) Preprocessing. (D) Superresolution mapping (Fig. 4).

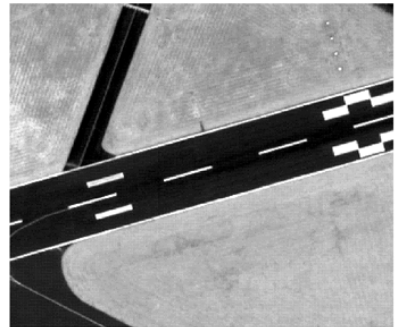

(a)

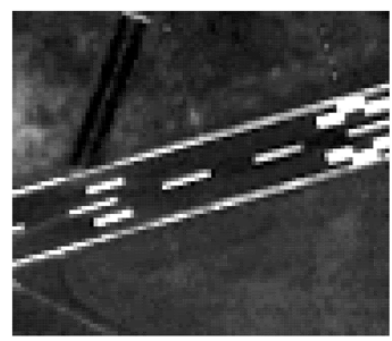

(c)

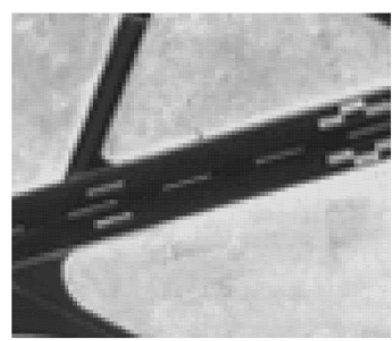

(e)

Fig. 11. (a) The $0.7-\mathrm{m}$ PAN image $(360 \times 300$ pixel $)$, (b) blue, (c) green, (d) red, and (e) NIR bands of $2.8-\mathrm{m}$ MS image $(90 \times 75)$.

\section{1) Training and Reference Data:}

Raw Data: A QuickBird MS image was acquired over an area of ChristChurch, UK on June 1, 2002. A subarea of PAN $(360 \times 300$ pixel $)$ [Fig. $11(a)]$ and MS $(90 \times 75$ pixel $)$

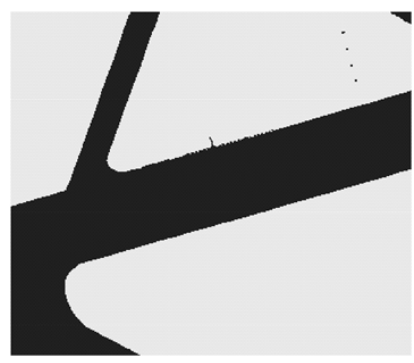

(a)

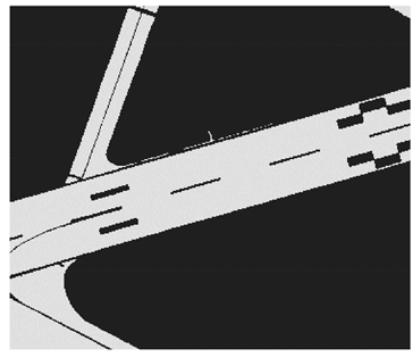

(c)

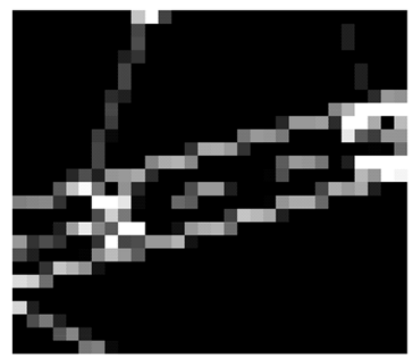

(e)

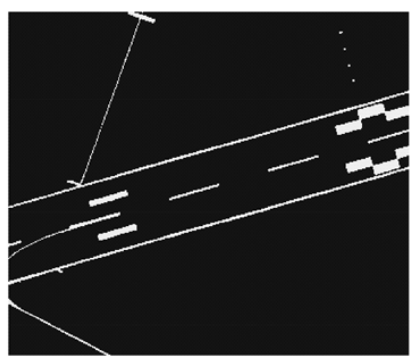

(b)

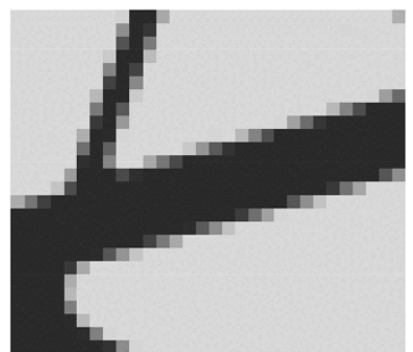

(d)

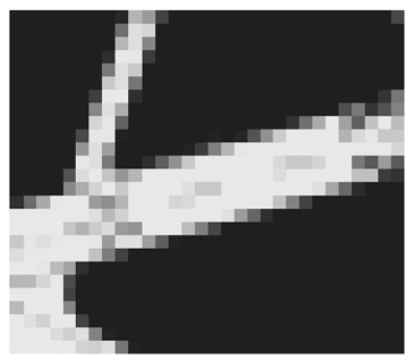

(f)
Fig. 12. Three land cover classes image for reference: (a) 0.7-m grass, (b) 0.7-m white surface, and (c) 0.7-m dark surface. Three land cover classes image for training: (d) 8.4-m grass, (e) 8.4-m white surface, and (f) 8.4-m dark surface.

[Fig. 11(b)-(e)] images was extracted from an area in the airport. Three land cover classes in the area were: grass, white surface and dark surface of the runway. The MS image was coregistered to the PAN image with a root mean square error of 0.25 pixels.

Training and Reference Data: Three land cover classes at $0.7-\mathrm{m}$ spatial resolution were obtained by manual digitising from the panchromatic image [Fig. 12(a)-(c)]. These land cover image were used as the reference data for the results of superresolution mapping. Thus, there was no image registration error of the PAN image against the reference data.

Superresolution methods use land cover proportions obtained by soft classification as input. To implement the soft classification, training data are required. In this paper, the soft classification was implemented at $8.4-\mathrm{m}$ spatial resolution. The training data, therefore, could be produced by degrading the land cover image at $0.7-\mathrm{m}$ spatial resolution by 12 times as in Fig. 12(d)-(f).

\section{2) Data Degradation:}

Multispectral Imagery $(8.4 \mathrm{~m})$ : The QuickBird MS image at 2.8- $\mathrm{m}$ spatial resolution was degraded by three times to produce a MS image at 8.4-m spatial resolution [Fig. 13(a)-(d)]. This MS image was then used to produce the land cover proportions at $8.4-\mathrm{m}$ spatial resolution using soft classification with 


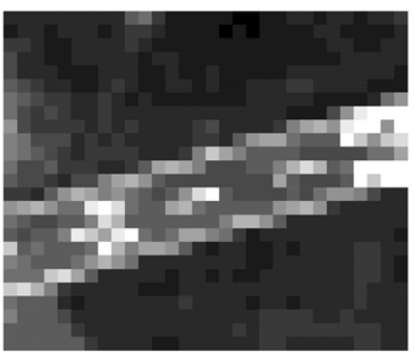

(a)

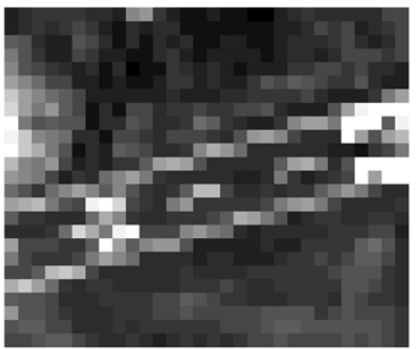

(c)

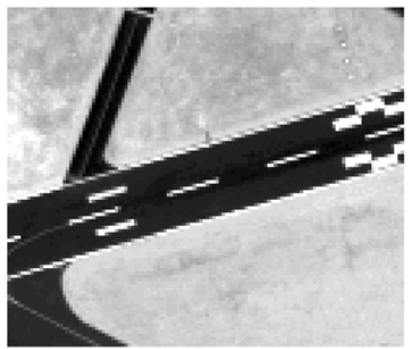

(e)

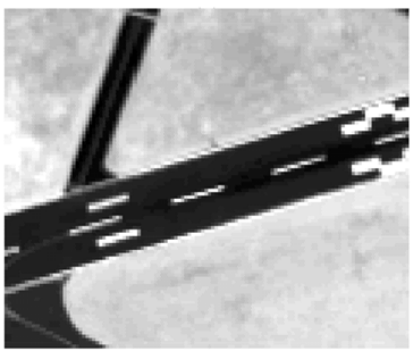

(g)

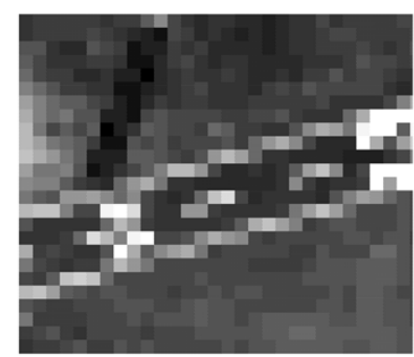

(b)

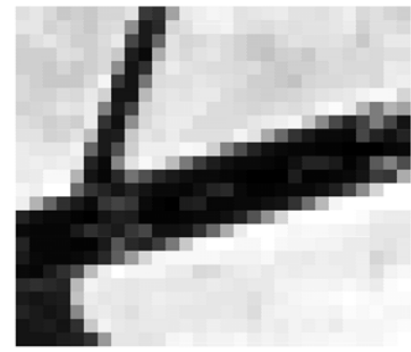

(d)

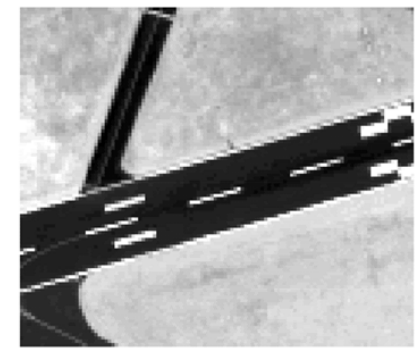

(f)

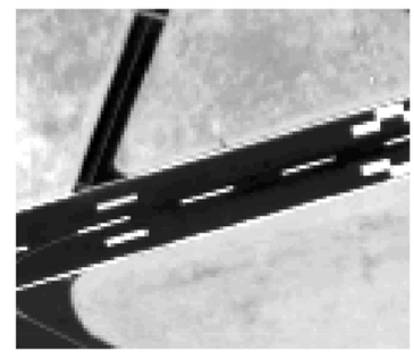

(h)
Fig. 13. Four bands (a) blue, (b) green, (c) red, and (d) NIR 8.4-m of simulated MS image. (e) The 2.1-m degraded PAN image. (f) The 0.5-pixel image registration error PAN image at $2.1-\mathrm{m}$ spatial resolution. (g) The 1-pixel image registration error PAN image at $2.1-\mathrm{m}$ spatial resolution and 1.5 -pixel image registration error PAN image at $2.1-\mathrm{m}$ spatial resolution.

training data in Fig. 12. The land cover proportions were then used to produce a $0.7-\mathrm{m}$ land cover image using superresolution mapping and the results were compared with the reference data in Fig. 12.

Panchromatic Imagery $(2.1 \mathrm{~m})$ : The 2.1-m PAN image was produced by degrading the $0.7-\mathrm{m}$ PAN image by three times [Fig. 13(e)]. The PAN image in Fig. 13(e) contained no image registration error. To evaluate the effect of the image registration error on the accuracy of the resulting land cover map, the PAN image was geocoded with root mean square errors (RMS) of 0.5 pixels [Fig. 13(f)], 1 pixel [Fig. 13(g)], and 1.5 pixels [Fig. 13(h)]. The proposed algorithm was then tested using the fused images obtained from these geocoded PAN images and the results were compared with the fused images without image registration error.

\section{3) Preprocessing:}

Fused Imagery $(2.1 \mathrm{~m})$ : Amongst the four fused spectral bands, three were used in the experiment (blue, green, and red). From the 2.1-m degraded PAN image [Fig. 13(e)] and 8.4-m MS image [Fig. 13(a)-(d)], the 2.1-m fused image was obtained using the Gram-Schmidt Spectral Sharpening method. To include the image registration error in the fused image, the PAN images with image registration RMS error in the range from 0.5 pixels to 1.5 pixels [Fig. 13(f)-(h)] were fused with the MS image to evaluate the effect of the image registration error on the algorithms.

Land Cover Proportion Imagery $(8.4 \mathrm{~m})$ : Proportion images at 8.4-m spatial resolution were produced from the 8.4-m MS image in Fig. 12 using the training data in Fig. 10. Obviously, this set of proportion images contains a certain amount of error including the MS image registration error. The predicted land cover proportions were then used for hard-classification, traditional superresolution mapping and the new method for superresolution mapping using the fused image. The error of the proportion predicting process has an impact on the results of all these methods. That means that the PAN image registration error is the only source of image registration error affecting the accuracy of superresolution mapping.

A $k$-nearest neighbor classifier ( $k$-NN) [8] was used for soft classification with $k=5$. The land cover proportion image was produced with overall area error proportion of $0.0242 \%$ and overall RMS error of 0.018159 pixels [13]. The proportions of three land cover classes can be seen in Fig. 14(a)-(c).

\section{B. Results}

In the experiment using degraded QuickBird imagery, the results produced by the three approaches were compared, as in the simulated data case. The 8.4-m hard-classified land cover map was obtained from the land cover proportion image [Fig. 14(a)-(c)] by assigning each 8.4-m pixel to the class of the largest proportion [Fig. 14(d)-(f)]. The HNN superresolution mapping by Tatem et al. [14] was based on the clustering goal functions, proportion constraint and multiclass constraint. Applying this HNN superresolution mapping approach, a $0.7-\mathrm{m}$ spatial resolution land cover map of three land cover classes was produced using the 8.4-m predicted land cover proportion image as input to the HNN superresolution mapping technique, with a zoom factor of 12 . After 6000 iterations with the weighting constants of $k_{1}=70, k_{2}=70, k_{3}=100$, and $k_{4}=70$, three land cover images were superresolved as in Fig. 14(g)-(i). Accuracy statistics for each class based on KIA, overall accuracy, and per-class omission and commission errors were presented to evaluate the predicted subpixel spatial resolution map (Table III).

The new HNN superresolution mapping technique was constrained by the 8.4-m land cover class proportion image and the $2.1-\mathrm{m}$ fused images. To estimate the local endmember spectra of three land cover classes, the 8.4-m land cover proportion image was used in combination with the 8.4-m MS images which were degraded from the $2.1-\mathrm{m}$ fused images. Based on the results of the simulated IKONOS data case, the weight value of 14 was used to determine the local endmember spectra. A zoom 


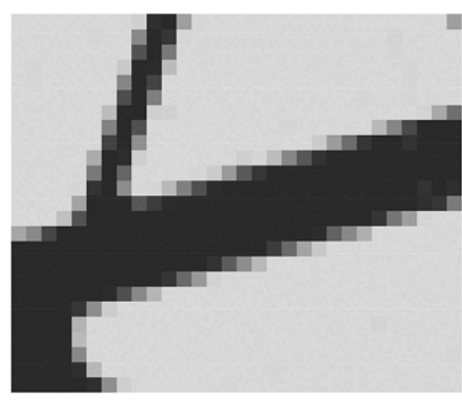

(a)

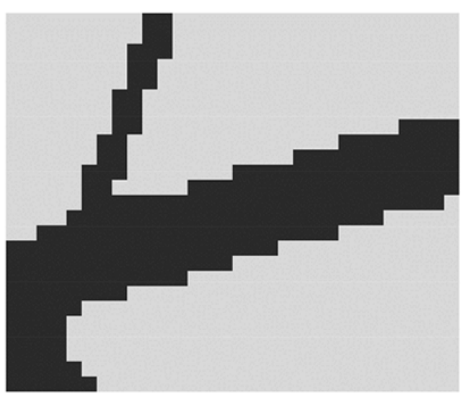

(d)

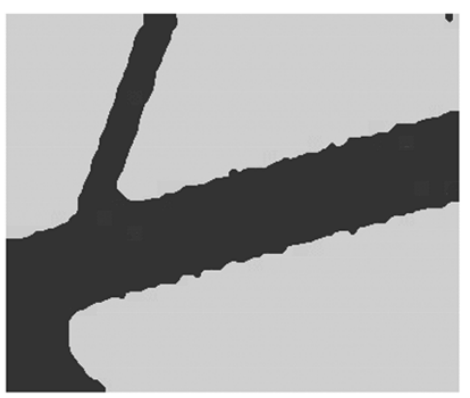

(g)

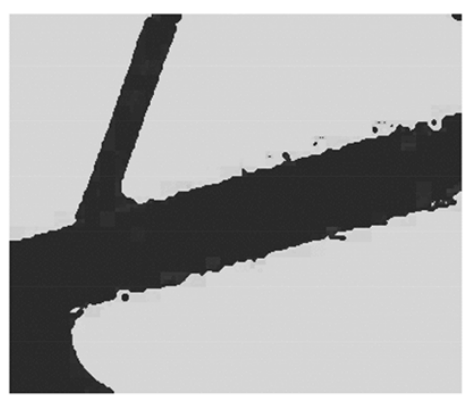

(j)

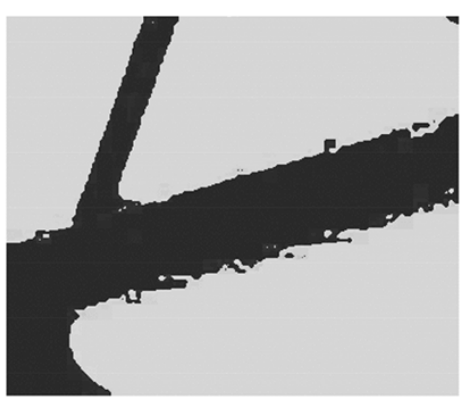

(m)

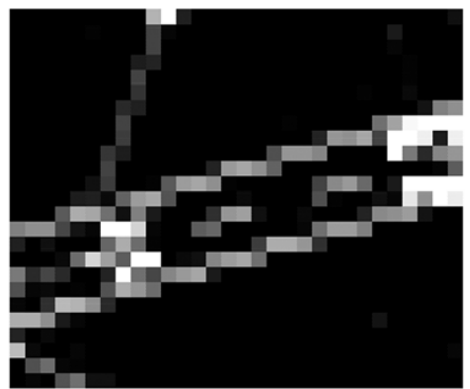

(b)

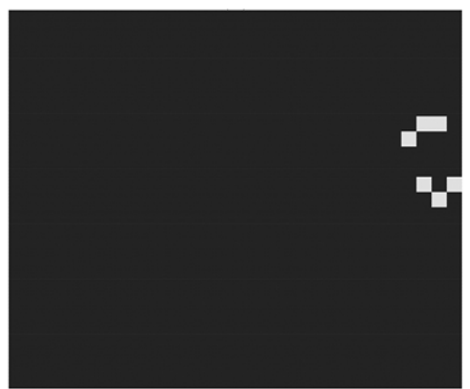

(e)

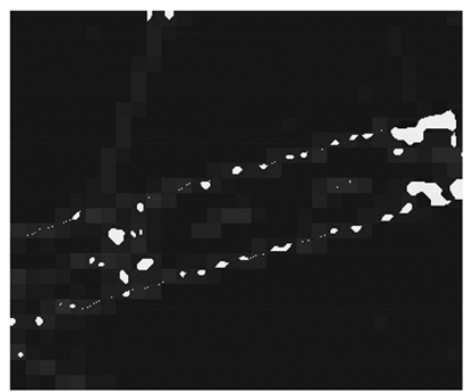

(h)

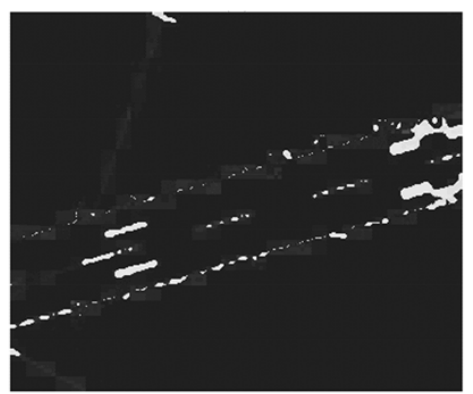

(k)

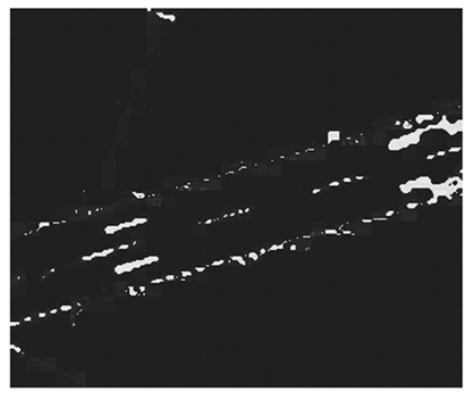

(n)

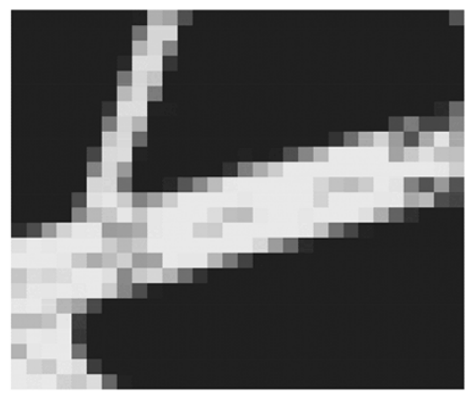

(c)

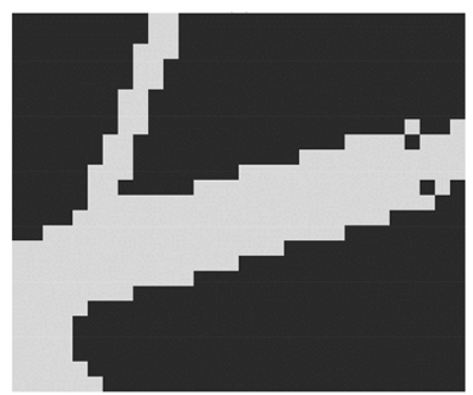

(f)

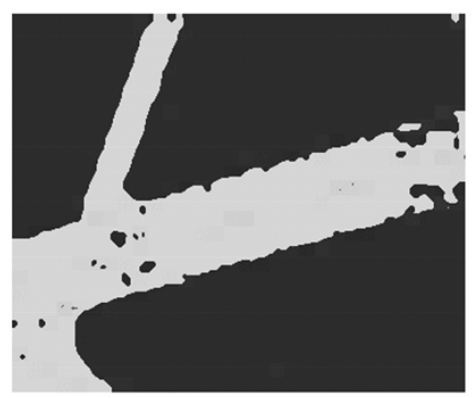

(i)

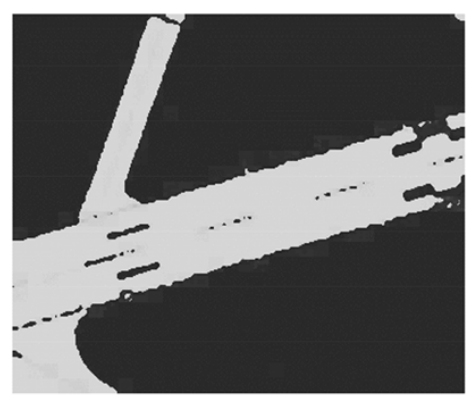

(1)

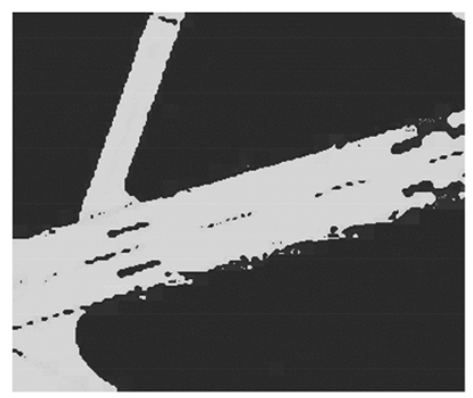

(o)

Fig. 14. (a) Grass, (b) White surface, and (c) Dark surface 8.4-m land cover proportion image. (d) Grass, (e) White surface, and (f) Dark surface 8.4-m hard-classified land cover image. (g) Grass, (h) White surface, and (i) Dark surface 0.7-m HNN superresolution mapping image. (j) Grass, (k) White surface, and (i) Dark surface 0.7-m HNN superresolution mapping using the fused image without image registration error. (m) Grass, (n) White surface, and (o) Dark surface 0.7 -m HNN superresolution mapping using the fused image with RMS image registration error of 1 pixel. 
TABLE III

ACCURACY STATISTICS OF DEGRADED QUICKBIRD RESUlTS

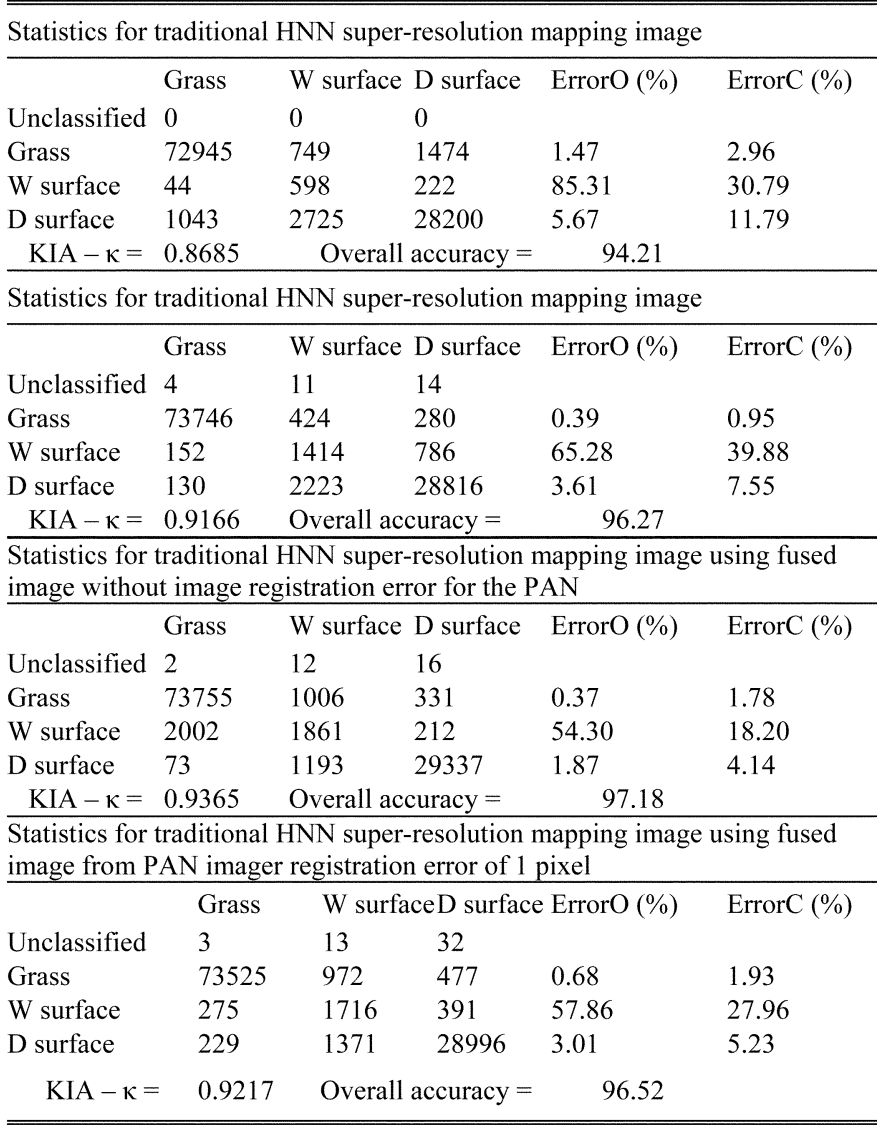

factor of 12 was used to produce a $0.7-\mathrm{m}$ spatial resolution map. With 6000 iterations and weighting constants of $k_{1}=70, k_{2}=$ $70, k_{3}=100, k_{4}=70$, and $k_{5}=100$, the HNN network using the fused MS image without PAN image registration error and the local endmember spectra produced the land cover images in Fig. 14(j)-(1). Using the same weighting constants with the fused image obtained from the PAN image with image registration RMS error of one pixel (the accuracy can be obtained normally in the geometric correction process) the HNN produced the land cover maps as in Fig. 14(m)-(o). Accuracy statistics of the predicted land cover map are given in Table III.

In comparison with the hard classification and the traditional HNN superresolution mapping, the resulting land cover map produced by the HNN using the fused image is visually more accurate. Similar to the simulated IKONOS dataset, the greatest improvement can be seen in the white surface class, where almost all subpixels belong to small objects. Without information from the fused image, the white surface subpixels of the linear objects in Fig. 12(b) were clustered into larger objects to satisfy the HNN goal functions as in Fig. 14(h). Although the fused image contains error due to the image registration error of the PAN image, the small and linear white surface objects can still be mapped and their shapes look similar to those in the reference image. This fact suggests that the new technique can be used for applications such as target identification.

The accuracy statistics showed a considerable increase in accuracy with the new technique. Overall accuracy of the land

\section{Effect of image registration error}

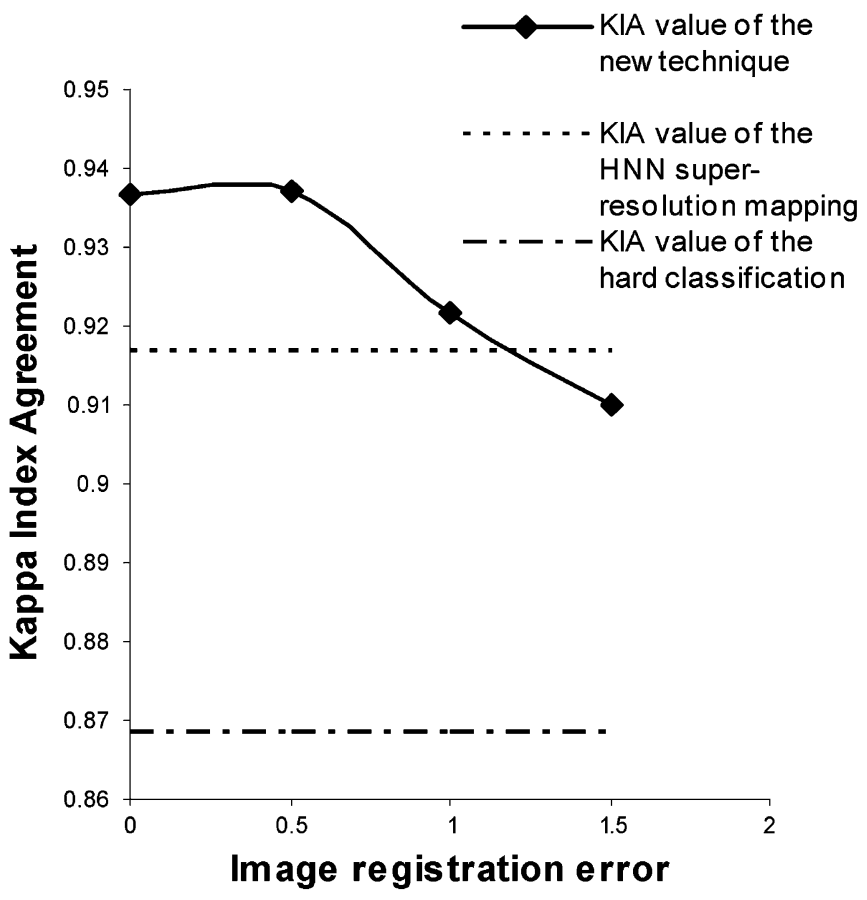

Fig. 15. Effect of the image registration on KIA value of resulted subpixel map using the HNN superresolution mapping using the fused image.

cover map increased by around 3\% from $94.21 \%$ for the hard classification to $97.18 \%$ for the superresolution mapping using the fused image without image registration error. With the fused image produced by the PAN image with image registration error of 1 pixel, the accuracy of the resulting $0.7-\mathrm{m}$ land cover map increased around $2 \%$ and $0.5 \%$ in comparison with the results of hard classification and the HNN superresolution mapping technique, respectively. The KIA value increased from 0.8685 for the hard-classified map and 0.9166 for the traditional HNN subpixel map to 0.9365 for the superresolution mapping using fused image without PAN image registration error and 0.9166 for the superresolution mapping using fused image with PAN image registration error of 1 pixel. Comparing with the resulting subpixel map produced by the HNN when the fused image was not used, the accuracy of the thematic map produced by the new technique (without PAN image registration error) increased approximately $1 \%$ in terms of overall accuracy.

Similar to the experiment involving simulated IKONOS imagery, amongst the three land cover classes, the accuracy of the white surface class increased most with the omission error reduced from $85.31 \%$ for the hard-classified image and $65.28 \%$ for the traditional HNN superresolution mapping to approximately 54\% and $57.85 \%$ for the new HNN superresolution mapping technique with and without PAN image registration error, respectively. The commission error reduced from $30.79 \%$ and $39.88 \%$ to $18.20 \%$ and $27.96 \%$, respectively after using the fused image. The increase in accuracy of the other two classes was not as great as that of the white surface class since most subpixels in these classes were grouped into larger objects.

To determine the effect of the image registration error on the results of the new technique, a series of PAN images with image registration error ranging from 0.5 pixels to 1.5 pixels were used 
to produce the fused images. Accuracy evaluation of the resulting land cover maps using these fused images based on the KIA value was implemented and presented in the plot in Fig. 15. Obviously, the plot shows that the KIA value reduced as the RMS image registration error increased. When the RMS image registration error increased to 1.5 pixels, the resulting subpixel map predicted by the new technique was less accurate than the results of the HNN without using the fused image. However, with the fused image produced from a PAN image with RMS error of one pixel (the accuracy of image registration that can be usually obtained in geometric correction of remotely sensed images) the accuracy of the subpixel map produced by the HNN superresolution mapping using the fused image was greater than that produced by the HNN superresolution mapping technique without using the fused image. It is recommended that the new technique should be used only if the PAN image is registered with an RMS error equal or smaller than one pixel.

\section{CONCLUSION}

This paper introduces the use of fused images for superresolution mapping. Data from the fused images were incorporated into the HNN optimization using forward and inverse models in the form of the reflectance constraint. The value of the constraint was calculated based on a linear mixture model, which used both global and local endmember spectra. The effectiveness of the technique was examined using both: 1) simulated IKONOS dataset and 2) a degraded QuickBird image (with and without image registration error). In both cases, the proportions images were supplemented by a simulated fused image and original MS image. The accuracy evaluation was implemented based on the KIA, overall accuracy, and omission and commission errors.

The results demonstrated that fused images can be used as a source of supplementary information for the HNN to predict land cover accurately at subpixel spatial resolution from simulated and real land cover proportion images. The analysis demonstrated a considerable increase in accuracy with the new technique, particularly for land cover features at the subpixel scale. For larger features, the technique increased the accuracy slightly. In addition, visual inspection of the resulting image showed pleasing improvements. The analysis also suggests that the new technique can be applied only if the RMS image registrion error of the PAN image is equal to or smaller than 1 pixel.

The result of the experiments suggest the potential for combining image fusion and superresolution mapping processes for real data. Thus, future research will develop a HNN to incorporate directly real panchromatic imagery as supplementary data to increase the accuracy and detail of the predicted subpixel land cover map.

\section{REFERENCES}

[1] R. J. Birk, T. Stanley, G. I. Snyder, T. A. Hennig, M. M. Fladeland, and F. Policelli, "Government programs for research and operational uses of commercial remote sensing data," Remote Sens. Environ., vol. 88, no. 1-2, pp. 3-16, 2003.

[2] J. B. Campell, Introduction to Remote Sensing, 2nd ed. London, U.K.: Taylor \& Francis, 1996.

[3] R. A. Schowengerdt, Remote Sensing: Models and Methods for Image Processing. San Diego, CA: Academic, 1997.
[4] J. J. Settle and N. A. Drake, "Linear mixing and the estimation of ground cover proportions," Int. J. Remote Sens., vol. 14, pp. 1159-1177, 1993.

[5] L. Bastin, "Comparison of fuzzy c-means classification, linear mixture modeling and MLC probabilities as tools for unmixing coarse pixels," Int. J. Remote Sens., vol. 18, pp. 3629-3648, 1997.

[6] G. M. Carpenter, S. Gopal, S. Macomber, S. Martens, and C. E. Woodcock, "A neural network method for mixture estimation for vegetation mapping," Remote Sens. Environ., vol. 70, pp. 138-152, 1999.

[7] G. M. Foody, R. M. Lucas, P. J. Curran, and M. Honzak, "Non-linear mixture modeling without end-members using an artificial neural network,” Int. J. Remote Sens., vol. 18, no. 4, pp. 937-953, 1997.

[8] R. A. Schowengerdt, "On the estimation of spatial-spectral mixing with classifier likelihood functions," Pattern Recognit. Lett., vol. 17, no. 13, pp. $1379-1387,1996$.

[9] M. Brown, H. Lewis, and S. Gunn, "Linear spectral mixture models and support vector machines for remote sensing," IEEE Trans. Geosci. Remote Sens., vol. 38, no. Sep., pp. 2346-2360, 2000.

[10] P. M. Atkinson, "Mapping sub-pixel boundaries from remotely sensed images," Innov. GIS, vol. 4, pp. 166-180, 1997.

[11] P. Aplin and P. M. Atkinson, "Sub-pixel land cover mapping for per-field classification," Int. J. Remote Sens., vol. 22, pp. 2853-2858, 2001.

[12] J. Verhoeye and R. De Wulf, "Land cover mapping at sub-pixel scales using linear optimization techniques," Remote Sens. Environ., vol. 79, no. 1, pp. 96-104, 2002.

[13] A. J. Tatem, H. G. Lewis, P. M. Atkinson, and M. S. Nixon, "Super-resolution target identification from remotely sensed images using a Hopfield neural network," IEEE Trans. Geosci. Remote Sens., vol. 39, no. 4, pp. 781-796, Apr. 2001.

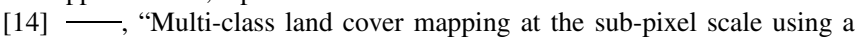
Hopfield neural network," Int. J. Appl. Earth Obs. Geoinf., vol. 3, no. 2 , pp. 184-190, 2001.

[15] - "Super-resolution land cover pattern prediction using a Hopfield neural network," in Uncertainty in Remote Sensing and GIS, G. M. Foody and P. M. Atkinson, Eds. Chichester, U.K.: Wiley, 2002, pp. 59-76.

[16] — , "Super-resolution land cover pattern prediction using a Hopfield neural network," Remote Sens. Environ., vol. 79, no. 1, pp. 1-14, 2002.

[17] A. J. Tatem, "Super-resolution land cover mapping from remotely sensed imagery using a Hopfield neural network," Ph.D. thesis, Univ. Southampton, Southampton, U.K., 2002.

[18] P. M. Atkinson, "GeoENV IV: Super-resolution land cover classification using geostatistical optimization," in Geostatistics for Environmental Applications, X. Sanchez-Villa, Ed. Dordrecht, The Netherlands: Kluwer, 2003.

[19] K. C. Mertens, L. P. C. Verbeke, E. I. Ducheyne, and R. R. De Wulf, "Using genetic algorithms in sub-pixel mapping," Int. J. Remote Sens., vol. 24, no. 21, pp. 4241-4247, 2003.

[20] K. C. Mertens, L. P. C. Verbeke, T. Westra, and R. R. De Wulf, "Subpixel mapping and sub-pixel sharpening using neural network predicted wavelet coefficients," Remote Sens. Environ., vol. 91, no. 2, pp. 225-236, 2004.

[21] W. Schneider and J. Steinwendner, "Land cover mapping by interrelated segmentation and classification of satellite images," Int. Arch. Photogramm. Remote Sens., pt. 7-4-3, vol. 32, 1999.

[22] C. Pinilla Ruiz and F. J. Ariza Lopez, "Restoring SPOT images using PSF-derived deconvolution filters," Int. J. Remote Sens., vol. 23, no. 12, pp. 2379-2391, 2003.

[23] J. W. Carper, T. M. Lillesand, and R. W. Kiefer, "The use of intensity-hue-saturation transformations for merging spot panchromatic and multispectral image data," Photogramm. Eng. Remote Sens., vol. 56, no. 4, pp. 459-467, 1990.

[24] P. S. Chavez, S. C. Sides, Jr, and J. A. Anderson, "Comparison of three different methods to merge multiresolution and multispectral data: Landsat TM and SPOT panchromatic," Photogramm. Eng. Remote Sens., vol. 57, no. 3, pp. 459-467, 1991.

[25] T. Ranchin, B. Aiazzi, L. Alparone, S. Baronti, and L. Wald, "Image fusion-The ARSIS concept and some successful schemes," ISPRS J. Photogramm. Remote Sens., vol. 58, pp. 4-18, 2003.

[26] C. A. Laben and B. V. Brower, "Process for enhancing the spatial resolution of multispectral imagery using pan-sharpening," U.S. Patent $6011875,2000$.

[27] C. K. Munechika, J. S. Warnick, C. Salvaggio, and J. R. Schott, "Resolution enhancement of multispectral image data to improve classification accuracy," Photogramm. Eng. Remote Sens., vol. 59, no. 1, pp. 67-72, 1993. 
[28] L. Wald, T. Ranchin, and M. Mangolini, "Fusion of satellite images of different spatial resolutions: Assessing the quality of resulting images," Photogramm. Eng. Remote Sens., vol. 63, no. 6, pp. 691-699, 1997.

[29] A. Schackelford and C. H. Davis, "A hierarchical fuzzy classification approach for high-resolution multispectral data over urban areas," IEEE Trans. Geosci. Remote Sens., vol. 41, no. 9, pp. 1920-1932, Sep. 2003.

[30] G. M. Foody, "Sharpening fuzzy classification output to refine the representation of sub-pixel land cover distribution," Int. J. Remote Sens., vol. 19, no. 13, pp. 2593-2599, 1998.

[31] H. Gross and J. R. Schott, "Application of spectral mixture analysis and image fusion techniques for image sharpening," Remote Sens. Environ., vol. 63, pp. 85-94, 1998.

[32] G. D. Robinson, H. N. Gross, and J. R. Schott, "Evaluation of two applications of spectral mixing models to image fusion," Remote Sens. Environ., vol. 71, no. 2, pp. 272-281, 2000.

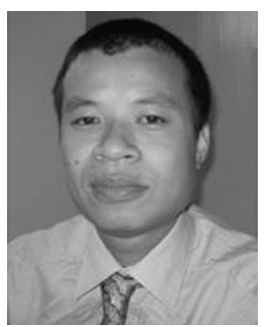

Minh Q. Nguyen was born May 13, 1972. He received the B.Sc. degree in engineering surveying from Hanoi University of Mining and Geology, Hanoi, Vietnam, and the M.Sc. degree from Seoul National University, Seoul, Korea, in 1993 and 1999, respectively. His M.Sc. thesis was on the application of GIS technique for land evaluation in Vietnam. $\mathrm{He}$ is currently pursuing the Ph.D. degree at the University of Southampton, Southampton, U.K.

From 1993 to 1996, he was with the Department of Engineering Surveying, Faculty of Geodesy, Surveying, and Cartography, Hanoi University of Mining and Geology, and was a Lecturer in engineering surveying there 1999 to 2003. His current research interests include land cover classification and image processing.

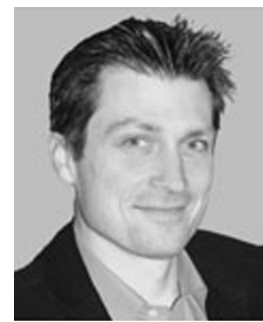

Peter M. Atkinson received the B.Sc. degree in geography from the University of Nottingham, Notthingham, U.K., and the Ph.D. degree in optimal sampling strategies for remote sensing from the University of Sheffield, Sheffield, U.K., (with Rothampsted Experimental Station) in 1986 and 1990 , respectively.

He is currently a Professor with the University of Southampton, Southampton, U.K. He was a Remote Sensing Anlyst with Logica from 1989 to 1990 . From 1990-1993, he was a PDRA with the University of Bristol, Bristol, U.K. He was a Lecturer with the University of Southampton from 1994 to 1999. He is a Coeditor for the International Journal of Remote Sensing Letters.

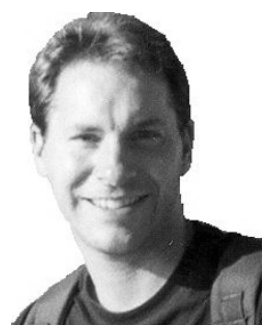

Hugh M. Lewis completed the B.Eng. degree in control engineering in 1992 and the M.Sc. degree in control systems from the University of Sheffield, Sheffield, U.K., and the Ph.D. degree from the University of Southampton, Southampton, U.K., in 1992, 1993, and 1999, respectively, for reseach on remote sensing of clouds using neural networks.

Between 1997 and 1999, he was a Research Fellow in the Department of Electronics and Computer Science and the Department of Aeronautics and Astronautics. During that time, he was a member of the FLIERS project investigating neural networks and empirical modeling techniques for land area estimation from remotely sensed imagery. In October 1999 he became a Research Fellow in the School of Engineering Sciences investigating the long-term evolution of space debris in geostationary Earth orbit. Between November 2002 and March 2003, he worked in the Intelligence, Agents, Multimedia Group in the Department of Electronics and Computer Science, on a project called MIAKT. This project looked at knowledge technologies to support medical diagnoses. In April 2003, he was appointed as a Lecturer in aerospace engineering in the School of Engineering Sciences. His research interests currently include neural networks for remote sensing, empirical data modeling, space debris and near-Earth object modeling, face recognition, and advanced knowledge technologies. 